\title{
Symmetries of quantum evolutions
}

\author{
Giulio Chiribella $\odot,{ }^{1,2,3, *}$ Erik Aurell, ${ }^{4, \dagger}$ and Karol Życzkowski ${ }^{5,6,+}$ \\ ${ }^{1}$ QICI Quantum Information and Computation Initiative, Department of Computer Science, \\ The University of Hong Kong, Pokfulam Road, Hong Kong 999077 \\ ${ }^{2}$ Department of Computer Science, University of Oxford, Wolfson Building, Parks Road, Oxford OX1 3QD, United Kingdom \\ ${ }^{3}$ Perimeter Institute for Theoretical Physics, 31 Caroline Street North, Waterloo, Ontario N2L 2Y5, Canada \\ ${ }^{4}$ KTH - Royal Institute of Technology, AlbaNova University Center, SE-106 91 Stockholm, Sweden \\ ${ }^{5}$ Institute of Theoretical Physics, Jagiellonian University, 30-348 Kraków, Poland \\ ${ }^{6}$ Center for Theoretical Physics, Polish Academy of Science, Al Lotników 32/44, 02-668 Warszawa, Poland
}

(Received 25 January 2021; revised 25 May 2021; accepted 27 May 2021; published 6 July 2021)

\begin{abstract}
A cornerstone of quantum mechanics is the characterization of symmetries provided by Wigner's theorem. Wigner's theorem establishes that every symmetry of the quantum state space must be either a unitary transformation or an antiunitary transformation. Here we extend Wigner's theorem from quantum states to quantum evolutions, including both the deterministic evolution associated with the dynamics of closed systems and the stochastic evolutions associated with the outcomes of quantum measurements. We prove that every symmetry of the space of quantum evolutions can be decomposed into two state space symmetries that are either both unitary or both antiunitary. Building on this result, we show that it is impossible to extend the time-reversal symmetry of unitary quantum dynamics to a symmetry of the full set of quantum evolutions. Our no-go theorem implies that any time-symmetric formulation of quantum theory must either restrict the set of the allowed evolutions or modify the operational interpretation of quantum states and processes. Here we propose a time-symmetric formulation of quantum theory where the allowed quantum evolutions are restricted to a suitable set, which includes both unitary evolution and projective measurements but excludes the deterministic preparation of pure states. The standard operational formulation of quantum theory can be retrieved from this time-symmetric version by introducing an operation of conditioning on the outcomes of past experiments.
\end{abstract}

DOI: 10.1103/PhysRevResearch.3.033028

\section{INTRODUCTION}

Symmetries play a central role in the modern approach to quantum mechanics $[1,2]$. They provide powerful methods for solving problems in atomic physics, condensed matter, high-energy physics, and quantum information science [3-5]. In addition, they offer guidance to the construction of new physical theories, such as Yang-Mills theories and other theories of fundamental interactions [6]. The starting point of all these investigations is Wigner's theorem [2,7], which characterizes the symmetries of the state space of any given quantum system.

In general, a symmetry is a one-to-one transformation that preserves a certain structure. For example, canonical transformations are symmetries in Hamiltonian mechanics, because they preserve the form of the Hamilton's equations. Wigner's

\footnotetext{
*giulio@cs.hku.hk

†eaurell@kth.se

${ }^{\ddagger}$ karol.zyczkowski@uj.edu.pl
}

Published by the American Physical Society under the terms of the Creative Commons Attribution 4.0 International license. Further distribution of this work must maintain attribution to the author(s) and the published article's title, journal citation, and DOI. theorem refers to the symmetries of the set of quantum states of a given quantum system. The structure of interest here is the probabilistic structure of quantum theory: when a symmetry is applied, the statistics of measurement outcomes should not change.

The original formulation of Wigner's theorem refers to pure states. Wigner established that every symmetry of the set of pure states can be represented by a transformation that is either unitary or antiunitary. Wigner's theorem can be equivalently formulated in terms of mixed states, represented by density matrices. In this formulation, the theorem states that every symmetry of the set of density matrices is either a unitary transformation, of the form $\rho \mapsto U \rho U^{\dagger}$ for some unitary operator $U$, or an antiunitary transformation, of the form $\rho \mapsto U \rho^{T} U^{\dagger}$, where $U$ is still a unitary operator, and $\rho^{T}$ is the transpose of the density matrix $\rho$ with respect to a fixed but otherwise arbitrary basis.

In recent years, there has been a growing interest in the extension of static notions, associated to the quantum state space, to dynamical notions, associated to the space of quantum evolutions. A series of works characterized the possible transformations that map quantum evolutions into quantum evolutions, known as quantum supermaps [8-12]. A related notion was explored in Ref. [13], which characterized the transformations of a certain set of completely positive maps other than the set of quantum evolutions. Higher-order 
transformations were applied to the study of causality in quantum theory [14-17], to analyze extensions of quantum theory [13], quantum dynamical resource theories [18-27], and a variety of quantum information processing tasks, such as process tomography [28], cloning [29,30], learning [31,32], and other conversions of quantum evolutions [33-35]. The lifting from states to evolutions has also led to new dynamical analogues of the notions of quantum entanglement [36,37] and quantum coherence [27,38].

In this paper we characterize the dynamical symmetries of quantum theory by extending Wigner's theorem from quantum states to quantum evolutions. The paper contains three main contributions, highlighted in the next section. The first contribution is a complete characterization of the symmetries of the space of quantum evolutions. We provide a Wigner-like theorem showing that every symmetry of quantum evolutions can be decomposed into two symmetries of quantum states, these two symmetries being either both unitary or both antiunitary. The second contribution is a rigorous proof that the set of all quantum evolutions admits no time-reversal symmetry. The third contribution is a time-symmetric variant of the quantum framework, obtained by restricting the set of allowed quantum evolutions to a suitable subset that preserves the maximally mixed state.

The paper is organized as follows. In Sec. II, we provide an overview of the main results of the paper. In Sec. III, we review Wigner's theorem and provide its mixed state version. In Sec. IV, we introduce the notion of symmetry of quantum evolutions, and we derive a Wigner theorem for quantum evolutions. In Sec. V, we show that the set of all quantum operations is incompatible with time symmetry. A way around this no-go result is provided in Sec. VI, where we propose time-symmetric variant of quantum theory, obtained by restricting the set of allowed quantum evolutions. Other ways around the no go result are discussed in Sec. VII. Finally, the conclusions are provided in Sec. VIII.

\section{OVERVIEW OF THE MAIN RESULTS}

\section{A. Wigner's theorem for quantum evolutions}

Our first contribution is a complete characterization of the symmetries of the space of quantum evolutions. The most general evolutions allowed by quantum mechanics are the so-called quantum operations $[39,40]$, which include both the deterministic evolution associated to the dynamics of closed systems, and the stochastic evolutions associated to the outcomes of quantum measurements. Mathematically, a quantum operation is a linear, completely positive map $\mathcal{Q}$ transforming density matrices on an input Hilbert space $\mathcal{H}_{\text {in }}$ into (generally subnormalized) density matrices on an output Hilbert space $\mathcal{H}_{\text {out }}$. The map $\mathcal{Q}$ satisfies the trace-nonincreasing condition

$$
\operatorname{Tr}[\mathcal{Q}(\rho)] \leqslant \operatorname{Tr}[\rho],
$$

for every operator $\rho$ acting on $\mathcal{H}_{\text {in }}$. When $\rho$ is a density matrix, the trace $\operatorname{Tr}[\mathcal{Q}(\rho)]$ is interpreted as the probability that the quantum operation $\mathcal{Q}$ takes place on the state $\rho$ in a suitable experiment. If this probability is zero for every quantum state, then we say that the operation $\mathcal{Q}$ is impossible.

A symmetry of the set of quantum operations is any one-toone transformation that preserves the probabilistic structure,

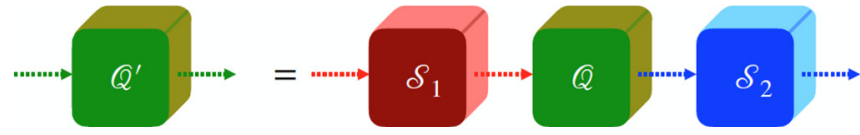

FIG. 1. Symmetry of quantum evolutions. The symmetry transformation turns a given quantum evolution $\mathcal{Q}$ into a new quantum evolution $\mathcal{Q}^{\prime}$, obtained by concatenating $\mathcal{Q}$ with two transformations of quantum states (denoted by $\mathcal{S}_{1}$ and $\mathcal{S}_{2}$ ), which are either both unitary or both antiunitary.

by (1) mapping random mixtures of quantum operations into random mixtures with the same probabilities and (2) mapping impossible operations into impossible operations.

We show that the symmetries of the full set of quantum operations have a very rigid structure: every symmetry of quantum operations can be broken down into two symmetries of quantum states, one symmetry for the input system, and one symmetry for the output system, as illustrated in Fig. 1. The two symmetries must be either both unitary, or both antiunitary, while all the other combinations are forbidden.

The Wigner theorem for quantum evolutions provides a rigorous framework for studying the dynamical symmetries in quantum theory. The theorem can be used to detect the presence or absence of certain symmetries, and to guide the formulation of new variants of quantum theory exhibiting some desired symmetry features, such as, e.g., time symmetry. The other contributions of our paper explore these two directions.

\section{B. No-go theorem for time-reversal symmetry}

Our second result is a proof that the set of all quantum evolutions is incompatible with time-reversal symmetry.

Microscopic time reversal is a cornerstone of quantum mechanics, and is inherited from the charge-time-parity symmetry of quantum field theory [41-43]. For closed quantum systems, the evolution is time-symmetric, in the sense that every unitary evolution $U$ admits a time reversal (for example, its inverse $U^{\dagger}$ or its transpose $U^{T}$ ), which is also a valid quantum evolution. A fundamental question is whether the time-reversal symmetry of unitary quantum dynamics can be extended to a symmetry of the set of all possible quantum evolutions.

In physics, symmetries are often viewed as changes of reference frame, and can be used to translate a description of phenomena made by one observer to a description of the same phenomena made of another observer [44]. Operationally, the question of time reversal is whether it is possible to find a "change of reference frame" that provides the description of a generic quantum evolution from the point of view of an observer whose time coordinate $t$ is replaced by $-t$. Of course, since most quantum evolutions are irreversible, the time reversal of a given evolution should not be expected to be a physical inversion. It could be an approximate inversion [45], a quasi-inversion [46,47], or, more generally, any evolution that is in one-to-one correspondence with the original evolution via a suitable change of description.

In our study of time symmetry, we focus on the set of quantum operations from a given quantum system to itself. In this case, the set of quantum operations includes the set 
of all unitary transformations. While the set of all unitary transformations is invariant under the standard time-reversal transformation $U \mapsto U^{\dagger}$, we show that such time reversal cannot be extended to the whole set of quantum operations. The same conclusion applies for the transformation $U \mapsto U^{T}$, which also cannot be extended to a transformation of the whole set of quantum operations.

Our result goes beyond the mere observation that the definition of quantum operation (1) is time-asymmetric because it makes a distinction between the input and the output. What we show is that it is in principle impossible to map the set of quantum operations into itself in a way that agrees with the transformation $U \mapsto U^{\dagger}$ (or with the transformation $U \mapsto U^{T}$ ) for all unitary evolutions.

Our general no-go theorem highlights the time-asymmetric nature of the set of general quantum evolutions, and provides insights into the problem of time symmetry in quantum theory [48-50], which has recently been the object of renewed interest in quantum foundations [51-57]. Our contribution to the debate is a rigorous definition of time symmetry of quantum evolutions, and a proof that the set of all quantum operations is incompatible with any notion of time symmetry that agrees with the probabilistic structure of quantum theory.

\section{A time-symmetric variant of quantum theory}

The last contribution of this paper is a candidate for a time-symmetric variant of quantum theory. We propose a restricted set of quantum evolutions, named time-symmetric quantum operations, which include unitary dynamics, and also all projective measurements described by von Neumann's and Lüders' update rules. Interestingly, our time-symmetric quantum operations also include all the state transformations allowed by the consistent history framework for closed quantum systems [58].

Crucially, our time-symmetric variant restricts the set of states that can be prepared deterministically: while it allows to prepare every quantum state with nonzero probability, the only state that can be prepared deterministically is the maximally mixed state.

The restriction to a single deterministic state is consistent with general considerations about causality in quantum theory. Quantum theory, in its standard operational formulation, satisfies the causality axiom [59-62], stating that it is impossible to send signals from the future to the past. In general, a physical theory satisfies the causality axiom if and only if, for every physical system described by the theory, there exists one and only one deterministic process that discards that system. The time reversal of the causality axiom, explored in Ref. [54], is that the theory contains only one deterministic state preparation for every system. It is natural to expect that a time-symmetric version of quantum theory should possess only a single deterministic preparation process and a single deterministic discarding process for every system. In this respect, our choice of time-symmetric quantum operations is a natural way to satisfy both conditions.

The standard quantum framework and its time-symmetric variant are different operational theories. In the standard framework, an agent can deterministically prepare any pure quantum state, while in the time-symmetric variant the agent can generate pure states with probability at most $1 / d$, where $d$ is the system's dimension. The difference affects which tasks can be implemented efficiently according to one theory or the other.

Despite the aforementioned difference, the standard operational framework of quantum theory can be retrieved from the time-symmetric version by introducing an operation of conditioning on state preparations. Operationally, the idea is that an agent who operates in the forward time direction can observe the preparation of a pure state, put the system aside, and store it in a memory for later use. Using a previously stored pure state, the agent can then implement any quantum operation on a given system, by letting the system interact with the memory via a suitable unitary dynamics. In this way, the full set of evolutions allowed in standard quantum theory is retrieved (see also the recent work by Hardy [57] for a similar argument, albeit with a different interpretation).

The idea that the standard quantum framework emerges from conditioning on state preparations is compatible with a few empirical observations. First, state preparations are often obtained by performing measurements, as in a Stern-Gerlach experiment. In those setups, the role of conditioning in the preparation of pure states (or more generally, nonmaximally mixed states) is immediately evident. Second, even those procedures that appear to deterministically generate pure states ultimately rely on some implicit form of conditioning on the outcomes of experiments done in the past. For example, the preparation of a ground state through successive cooling operations requires the experimenter to have access to low temperature reservoirs, corresponding to sets of particles in nearly pure states. Producing such reservoirs from scratch would require the ability to filter particles based on their quantum state, which again comes down to conditioning on the outcomes of certain quantum experiments. In this respect, our time-symmetric version of quantum theory is closely related to the resource theory of purity [63-65], where every deviation from the maximally mixed state is regarded as a resource.

The above considerations suggest that one could regard the time-symmetric variant as fundamental, and the standard quantum framework as an effective theory, describing the time-asymmetric abilities of a certain type of agents. This idea agrees with similar considerations made in a series of recent works [55-57], which reexamined the question of time symmetry in quantum theory from an operational perspective.

\section{SYMMETRIES OF QUANTUM STATES}

Here we first review the classic formulation of Wigner's theorem, and then provide an equivalent formulation in terms of mixed states.

\section{A. Wigner's theorem}

The original formulation of Wigner's theorem [2,7] is in terms of pure states. The pure states of a system with Hilbert space $\mathcal{H}$ are described by unit rays, that is, equivalence classes of unit vectors that are equal up to a global phase. Specifically, for a unit vector $\psi \in \mathcal{H}$, the corresponding unit ray is the set $\underline{\psi}:=\left\{e^{i \gamma} \psi \mid \gamma \in \mathbb{R}\right\}$. 
The outcome probabilities of basic quantum measurements are computed through Born's statistical formula. The fundamental quantity appearing in Born's formula is the ray product

$$
\underline{\psi} \cdot \underline{\phi}=|\langle\psi \mid \phi\rangle|
$$

whose value is independent of the choice of unit vectors $|\psi\rangle$ and $|\phi\rangle$ used to represent the sets $\psi$ and $\phi$, respectively.

A symmetry of the set of pure states is a one-to-one transformation that preserves the outcome probabilities in all possible experiments. Mathematically, the set of pure states is the so-called ray space $\mathcal{H}$, that is, the set of unit rays, equipped with the ray product. A symmetry is is a transformation of the ray space into itself, or, more generally, a transformation of a ray space $\underline{\mathcal{H}}$ into another ray space $\underline{\mathcal{K}}$.

Definition 1. A symmetry transformation from the ray space $\underline{\mathcal{H}}$ to the ray space $\underline{\mathcal{K}}$ (a ray space symmetry, for short) is a bijective function $\underline{S}: \underline{\mathcal{H}} \rightarrow \underline{\mathcal{K}}$ that preserves the ray product, namely

$$
\underline{S}(\underline{\psi}) \cdot \underline{S}(\underline{\phi})=\underline{\psi} \cdot \underline{\phi} \quad \forall \underline{\psi}, \underline{\phi} \in \underline{\mathcal{H}} .
$$

We recall that a bijective function $f$ from set $A$ to set $B$ is a transformation that maps distinct elements of $A$ into distinct elements of $B$, in such a way that every element of $B$ is obtained by applying $f$ to some element of A. Intuitively, the property of being bijective is necessary for the two ray spaces $\underline{\mathcal{H}}$ and $\underline{\mathcal{K}}$ to be regarded as different descriptions of the same physical system.

Wigner's theorem provides a complete characterization of the ray space symmetries. Specifically, it shows that every ray space symmetry is induced by a transformation on the underlying Hilbert space:

Theorem 1 (Wigner's theorem). For every ray space symmetry $\underline{S}: \underline{\mathcal{H}} \rightarrow \underline{\mathcal{K}}$ there exists a Hilbert space transformation $S: \mathcal{H} \rightarrow \mathcal{K}$ such that $(1) \underline{S}(\psi)=S(\psi)$ for every $\psi \in \mathcal{H}$ and (2) the transformation $S$ is either unitary or antiunitary.

We recall that a bijective transformation $S: \mathcal{H} \rightarrow \mathcal{K}$ is unitary if $\langle S(\psi) \mid S(\phi)\rangle=\langle\psi \mid \phi\rangle$ for every pair of vectors $\psi$ and $\phi$ in $\mathcal{H}$, and antiunitary if $\langle S(\psi) \mid S(\phi)\rangle=\langle\phi \mid \psi\rangle$.

The prototype of an antiunitary transformation is the transformation $S: \psi \rightarrow \psi^{*}$, where $\psi^{*}$ is the complex conjugate of the vector $\psi$ with respect to a fixed (but otherwise arbitrary) orthonormal basis. In general, the action of a generic antiunitary transformation $S$ can be represented as $S(\psi)=U \psi^{*}$, where $U: \mathcal{H} \rightarrow \mathcal{K}$ is a unitary operator. A general treatment of antiunitary transformations can be found in the recent work by Uhlmann [66].

\section{B. Density matrix formulation of Wigner's theorem}

Wigner's theorem can be equivalently formulated as a characterization of the symmetries of the full state space, including mixed states described by density matrices. In this case, the probabilistic interpretation of quantum states implies the possibility of considering random mixtures of different density matrices. Operationally, the randomization can be due to ignorance about the state of the quantum system, or also to an actual physical mechanism that lets the choice of state be controlled by some random event, such as the result of coin toss.
In the following, we will denote the set of all density matrices on the Hilbert space $\mathcal{H}$ by

$$
\operatorname{St}(\mathcal{H}):=\{\rho \in L(\mathcal{H}) \mid \rho \geqslant 0, \operatorname{Tr}[\rho]=1\},
$$

$L(\mathcal{H})$ denoting the set of linear operators on $\mathcal{H}$. A symmetry of state spaces is then defined to be as a one-to-one transformation that preserves random mixtures:

Definition 2. A symmetry transformation from the state space $\operatorname{St}(\mathcal{H})$ to the state space $\operatorname{St}(\mathcal{K})$ (a state space symmetry, for short) is a bijective transformation $\mathcal{S}: \operatorname{St}(\mathcal{H}) \rightarrow \operatorname{St}(\mathcal{K})$ that is consistent with randomizations, namely,

$$
\mathcal{S}(p \rho+(1-p) \sigma)=p \mathcal{S}(\rho)+(1-p) \mathcal{S}(\sigma),
$$

for every pair of density matrices $\rho$ and $\sigma$, and for every probability $p \in[0,1]$.

Wigner's original theorem is equivalent to the following statement:

Theorem 2. (Density matrix formulation of Wigner's theorem) Every state space symmetry $\mathcal{S}: \operatorname{St}(\mathcal{H}) \rightarrow \operatorname{St}(\mathcal{K})$ is either a unitary transformation, of the form $\mathcal{S}(\rho)=U \rho U^{\dagger}$ for some unitary operator $U: \mathcal{H} \rightarrow \mathcal{K}$, or an antiunitary transformation, of the form $\mathcal{S}(\rho)=U \rho^{T} U^{\dagger}$, where $\rho^{T}$ denotes the transpose of $\rho$ with respect to a fixed orthonormal basis.

Theorem 2 can be derived from the original Wigner's theorem, as shown in Appendix A. Conversely, it is also possible to show that theorem 2 implies the original Wigner's theorem, as shown in Appendix B. In summary, theorem 2 is an equivalent formulation of Wigner's original result in the language of density matrices.

\section{SYMMETRIES OF QUANTUM EVOLUTIONS}

In this section, we derive a Wigner theorem for quantum evolutions, showing that every symmetry of the set of quantum operations can be broken down into two state space symmetries, which are either both unitary or both antiunitary.

\section{A. Quantum operations}

Quantum operations $[39,40]$ provide a unified framework for treating all state evolutions in quantum mechanics. In general, a quantum state change can be stochastic, meaning that an initial state $\rho$ is transformed into a final state $\rho^{\prime}$ with some probability $p$, generally smaller than 1 . The nonunit probability of obtaining the output state can be accounted for by considering subnormalized states, corresponding to nonnegative operators $\rho$ with trace $\operatorname{Tr}[\rho] \leqslant 1$. The trace $\operatorname{Tr}[\rho]$ is then interpreted as the probability that the state is prepared by some suitable stochastic process. When such probability is nonzero, the state of the system conditional to the occurrence of the process is the normalized state $\rho / \operatorname{Tr}[\rho]$.

Mathematically, a quantum operation is a completely positive, trace-nonincreasing linear map $\mathcal{Q}$, transforming an initial state $\rho$ into a subnormalized state $\mathcal{Q}(\rho)$. The probability that the state change takes place on the state $\rho$ (in a stochastic process including the quantum operation $\mathcal{Q}$ among its possible events) is the trace $\operatorname{Tr}[\mathcal{Q}(\rho)]$. When such a state change occurs, the state of the system becomes $\rho^{\prime}=\mathcal{Q}(\rho) / \operatorname{Tr}[\mathcal{Q}(\rho)]$.

Kraus' theorem [39] shows that the action of a quantum operation $\mathcal{Q}$ on a generic density matrix $\rho$ can be expressed as 
$\mathcal{Q}(\rho)=\sum_{i} Q_{i} \rho Q_{i}^{\dagger}$, where the operators $\left(Q_{i}\right)$, called Kraus operators, satisfy the condition $\sum_{i} Q_{i}^{\dagger} Q_{i} \leqslant I_{\text {in }}$. When the equality condition $\sum_{i} Q_{i}^{\dagger} Q_{i}=I_{\text {in }}$ is satisfied, the quantum operation is called a quantum channel [67].

In the following, we will denote the set of all quantum operations by $\operatorname{Op}\left(\mathcal{H}_{\text {in }}, \mathcal{H}_{\text {out }}\right)$ and the subset of all quantum channels by $\operatorname{Chan}\left(\mathcal{H}_{\text {in }}, \mathcal{H}_{\text {out }}\right)$, where $\mathcal{H}_{\text {in }}$ and $\mathcal{H}_{\text {out }}$ are the Hilbert spaces of the input and output system, assumed to be finite dimensional for simplicity of presentation. We will call $\operatorname{Op}\left(\mathcal{H}_{\text {in }}, \mathcal{H}_{\text {out }}\right)$ and Chan $\left(\mathcal{H}_{\text {in }}, \mathcal{H}_{\text {out }}\right)$ the operation space and the channel space, respectively.

Like the quantum state space, the quantum operation space is convex, meaning that the random mixture of two quantum operations is also a quantum operation. Several aspects of the convex structure of the quantum operation space were investigated in Ref. [68].

The quantum operation space also contains a special element, namely the null quantum operation $\mathcal{Q}=0$, describing the limit case of a stochastic evolution that has zero probability to take place. Convexity and the presence of a null element are the two key elements of the probabilistic structure of the set of quantum operations.

\section{B. Symmetries of the set of quantum operations}

A symmetry of quantum operations is a reversible transformation that it is consistent with randomizations and maps impossible events into impossible events.

Definition 3. A symmetry transformation from the operation space $\operatorname{Op}\left(\mathcal{H}_{\text {in }}, \mathcal{H}_{\text {out }}\right)$ to the operation space $\operatorname{Op}\left(\mathcal{K}_{\text {in }}, \mathcal{K}_{\text {out }}\right)$ (an operation space symmetry, for short) is a bijective transformation $\mathcal{S}: \operatorname{Op}\left(\mathcal{H}_{\text {in }}, \mathcal{H}_{\text {out }}\right) \rightarrow \operatorname{Op}\left(\mathcal{K}_{\text {in }}, \mathcal{K}_{\text {out }}\right)$ that (1) is consistent with randomizations, namely, $\mathcal{S}(p \mathcal{Q}+(1-$ p) $\mathcal{R})=p \mathcal{S}(\mathcal{Q})+(1-p) \mathcal{S}(\mathcal{R})$, for every pair of quantum operations $\mathcal{Q}$ and $\mathcal{R}$, and for every probability $p \in[0,1]$, and (2) transforms the null operation of $\operatorname{Op}\left(\mathcal{H}_{\text {in }}, \mathcal{H}_{\text {out }}\right)$ into the null operation of $\operatorname{Op}\left(\mathcal{K}_{\text {in }}, \mathcal{K}_{\text {out }}\right)$.

It is not difficult to see that every symmetry of quantum operations should map quantum channels into quantum channels.

Proposition 1. For every operation space symmetry $\mathcal{S}: \operatorname{Op}\left(\mathcal{H}_{\text {in }}, \mathcal{H}_{\text {out }}\right) \rightarrow \operatorname{Op}\left(\mathcal{K}_{\text {in }}, \mathcal{K}_{\text {out }}\right), \quad$ one has $\mathcal{S}\left(\operatorname{Chan}\left(\mathcal{H}_{\text {in }}, \mathcal{H}_{\text {out }}\right)\right)=\operatorname{Chan}\left(\mathcal{K}_{\text {in }}, \mathcal{K}_{\text {out }}\right)$.

This result, proved in Appendix C, is conceptually important because it shows that every operation space symmetry induces a channel space symmetry.

\section{Wigner theorem for quantum operations}

We now provide a complete characterization of the symmetries of quantum operations, in analogy to Wigner's theorem for quantum states. Our result shows that every operation space symmetry can be decomposed into two state space symmetries, of which one transforms the input, and the other transforms the output.

Theorem 3. (Wigner's theorem for quantum operations) Every operation space symmetry $\mathcal{S}: \operatorname{Op}\left(\mathcal{H}_{\text {in }}, \mathcal{H}_{\text {out }}\right) \rightarrow$ $\operatorname{Op}\left(\mathcal{K}_{\text {in }}, \mathcal{K}_{\text {out }}\right)$ is of the form $\mathcal{S}(\mathcal{Q})=\mathcal{S}_{2} \circ \mathcal{Q} \circ \mathcal{S}_{1}$, where $\mathcal{S}_{1}$ : $\operatorname{St}\left(\mathcal{K}_{\text {in }}\right) \rightarrow \operatorname{St}\left(\mathcal{H}_{\text {in }}\right)$ and $\mathcal{S}_{2}: \operatorname{St}\left(\mathcal{H}_{\text {out }}\right) \rightarrow \operatorname{St}\left(\mathcal{K}_{\text {out }}\right)$ are state space symmetries that are either both unitary or both antiunitary.

The proof of Theorem 3 is provided in Appendix D, using technical lemmas established in Appendices E, F, and G. Like Wigner's original theorem, our result establishes that the possible symmetries are of two types. We call these two types double unitary symmetries and double antiunitary symmetries.

Double unitary symmetries can be physically implemented by inserting the input quantum operation between two reversible quantum processes. Such symmetries are compatible with an active interpretation, in which an agent implements the symmetry by engineering the system's dynamics $[8,9]$.

In contrast, double antiunitary transformations are not compatible with an active interpretation, at least not in standard quantum mechanics, where antiunitary operations are excluded from the set of allowed evolutions.

The prototype of a double antiunitary symmetry is the "double transpose" transformation $\tau_{\text {in/out }}: \mathcal{Q} \mapsto \mathcal{Q}^{\prime}=\tau_{\text {out }} \circ$ $\mathcal{Q} \circ \tau_{\text {in }}$, where $\tau_{\text {in }}\left(\tau_{\text {out }}\right)$ is the transpose on the input (output) system with respect to a fixed basis. In the Kraus representation, the double transpose corresponds to the complex conjugate of the Kraus operators, mapping a quantum operation with Kraus operators $\left\{Q_{i}\right\}$ into a new quantum operation with Kraus operators $\left\{Q_{i}^{*}\right\}$. The double transpose transformation appeared in the seminal work by Holevo and Werner on the capacity of bosonic Gaussian channels [69], where it was used to show that entanglement-breaking channels have zero quantum capacity. Approximate physical realizations of the double transpose were studied in Refs. [33-35,70], which focused on approximating the complex conjugation of unknown unitary dynamics.

In general, every double antiunitary transformation has the form $\mathcal{S}(\mathcal{Q})=\mathcal{U} \circ \tau_{\text {in } / \text { out }}(\mathcal{Q}) \circ \mathcal{V}$, where $\mathcal{U}: \operatorname{St}\left(\mathcal{K}_{\text {in }}\right) \rightarrow$ $\operatorname{St}\left(\mathcal{H}_{\text {in }}\right)$ and $\mathcal{V}: \operatorname{St}\left(\mathcal{H}_{\text {out }}\right) \rightarrow \operatorname{St}\left(\mathcal{K}_{\text {out }}\right)$ are unitary symmetries. In other words, the double transpose is the seed of all possible double antiunitary symmetries.

Further discussion on the differences between double unitary and double antiunitary symmetries, in relation to the notion of complete positivity, is provided in Appendix $\mathrm{H}$.

\section{NO TIME REVERSAL OF GENERAL QUANTUM EVOLUTIONS}

\section{A. Time reversal symmetry of unitary evolutions and bistochastic channels}

The set of unitary evolutions exhibits an obvious timereversal symmetry: for every unitary evolution $U$, the inverse matrix $U^{\dagger}$ is also unitary and describes a valid quantum evolution. At the level of quantum channels, the standard time-reversal maps the unitary channel $\mathcal{U}: \rho \mapsto U \rho U^{\dagger}$ into the inverse channel $\mathcal{U}^{\dagger}: \rho \mapsto U^{\dagger} \rho U$.

For certain irreversible quantum evolutions, it is still possible to define a meaningful notion of time reversal. Indeed, the time-reversal symmetry of the set of unitary channels can be uniquely extended to a larger set of quantum evolutions, known as the set of bistochastic (or doubly stochastic) quantum channels [71,72]. A bistochastic channel is a completely positive trace-preserving map $\mathcal{C}: \operatorname{St}(\mathcal{H}) \rightarrow \operatorname{St}(\mathcal{H})$ with the additional property that $\mathcal{C}(I)=I$. 
The set of bistochastic channels contains highly irreversible evolutions, such as the evolution that takes every quantum state to the maximally mixed state. For such evolutions, the time reversal is not the process that brings back the system to the initial state, for such a process cannot be defined without information about the environment with which the system interacted. Instead, the time reversal of a bistochastic channel describes how the same physical device would respond to a preparation of states in the future, rather than a preparation of states in the past [56]. To clarify this point, consider the qubit channel $\mathcal{C}_{0}: \rho \mapsto \mathcal{C}_{0}(\rho)=I / 2$, which maps every state to the maximally mixed state. This channel can be realized as a uniform random mixture of four unitary channels, corresponding to the four Pauli matrices $I, X, Y$, and $Z$. To be consistent with random mixtures, the time reversal of channel $\mathcal{C}_{0}$ must be the uniform mixture of the inverses of the four unitary channels, which again correspond to the four Pauli matrices $I, X, Y$, and $Z$. In summary, the time reversal of channel $\mathcal{C}_{0}$ is channel $\mathcal{C}_{0}$ itself: in this example, both an ordinary agent and an agent with an inverted time arrow would describe the overall input-output transformation as a channel that maps every state into the maximally mixed state.

The standard time reversal of the bistochastic channel $\mathcal{C}$ is the channel $\mathcal{C}^{\dagger}$ defined by $\mathcal{C}^{\dagger}(\rho)=\sum_{i} C_{i}^{\dagger} \rho C_{i}$. Note that, in general, the channel $\mathcal{C}^{\dagger}$ is not the inverse of the channel $\mathcal{C}$. In a sense, however, $\mathcal{C}^{\dagger}$ can be thought as an approximate inversion, known as Petz' recovery map [73], and has many applications in quantum information [74-79]. At the structural level, the transformation $\Theta: \mathcal{C} \mapsto \mathcal{C}^{\dagger}$ is a symmetry of the set of bistochastic channels: it is a bijective transformation, and it is compatible with randomizations.

The set of unitary evolutions also admits an alternative time reversal, given by the map $\mathcal{U} \mapsto \mathcal{U}^{T}$, where $\mathcal{U}^{T}$ is the transpose unitary channel, defined by $\mathcal{U}^{T}(\rho)=U^{T} \rho U^{*}[56]$. This alternative time reversal can also be extended uniquely to the set of bistochastic channels, by mapping the channel $\mathcal{C}$ into the channel $\mathcal{C}^{T}$ defined by $\mathcal{C}^{T}(\rho)=\sum_{i} C_{i}^{T} \rho C_{i}^{*}$. The alternative time reversal $\Theta^{\prime}: \mathcal{C} \mapsto \mathcal{C}^{T}$ is also a symmetry of the set of bistochastic channels: it is bijective and compatible with randomizations.

The maps $\Theta$ and $\Theta^{\prime}$ can be characterized as the two canonical time reversals on the set of bistochastic channel [56]. More information about their structure can be found in Appendix I, where we characterize the action of $\Theta$ and $\Theta^{\prime}$ in terms of the Choi representation [80] (see also Refs. [81] and [40]).

\section{B. No-go theorem for time-reversal symmetry of the set of quantum operations}

A natural question is whether the time-reversal symmetries of the set of bistochastic channels can be extended to symmetries of the whole set of quantum operations.

The obvious candidates of time reverals are the maps $\Theta: \mathcal{Q} \mapsto \mathcal{Q}^{\dagger}$ and $\Theta^{\prime}:=\mathcal{Q} \mapsto \mathcal{Q}^{T}$, now defined on arbitrary quantum operations $\mathcal{Q}$. It is relatively easy to see, however, that these two maps fail to be symmetries of the full set of quantum operations. In principle, however, there could exist other ways to extend the time reversal from the set of bistochastic channels to the set of all quantum operations. This possibility is ruled out by the following theorem.
Theorem 4. (No time reversal of arbitrary quantum operations) No symmetry $\mathcal{S}$ of the set of quantum operations satisfies the condition $\mathcal{S}(\mathcal{U})=\mathcal{U}^{\dagger}$ for every unitary channel $\mathcal{U}$, or the condition $\mathcal{S}(\mathcal{U})=\mathcal{U}^{T}$ for every unitary channel $\mathcal{U}$.

The proof can be found in Appendix J.

Theorem 4 shows that the structure of set of all quantum evolutions is fundamentally incompatible with time-reversal symmetry: informally, this means that it is impossible to define a "change of reference frame" corresponding to the transformation $t \mapsto-t$, and compatible with the probabilistic interpretation of quantum evolutions.

It is worth noting that our no-go result applies to the whole set of quantum operations. An interesting open question is whether our no-go theorem remains valid for the subset of quantum channels. A no-go theorem for time-reversal symmetries of quantum channels has been recently proven in Ref. [56], under the additional assumption that time reversal should reverse the order in which processes take place in any time sequence. We conjecture that this assumption can be lifted, and that the no-go theorem to time reversal applies also to symmetries of the set of quantum channels. Such extension of the no-go theorem, however, is beyond the scope of the present paper, which focusses on the symmetries of the full set of quantum evolutions.

Our no-go result on time reversal poses a strong constraint on any attempt to formulate quantum theory as a time-symmetric operational theory. To better understand the nature of this constraint, it is useful to consider possible relaxations of the theorem. In the following we discuss three such relaxations: the first relaxation consists in restricting the set of allowed quantum evolutions to a time-symmetric subset, the other two relaxations consist in weakening the notion of symmetry in a way that evades our no-go theorem.

\section{A TIME-SYMMETRIC VARIANT OF QUANTUM THEORY}

In this section, we provide a time-symmetric variant of quantum theory wherein the allowed channels transform maximally mixed states into maximally mixed states. We show that the standard formulation of quantum theory can be obtained from the time-symmetric variant through a suitable operation of conditioning. Then, we show that the timesymmetric variant is maximal among the time-symmetric quantum theories in which all unitary channels are regarded as allowed dynamics.

\section{A. Time-symmetric quantum operations}

One way to circumvent our no-go theorem on time symmetry is to restrict the attention to a subset of quantum evolutions on which a time-reversal symmetry can be defined. Here we propose the set of quantum operations satisfying the conditions

$$
\mathcal{Q}^{\dagger}\left(I_{\text {out }}\right) \leqslant I_{\text {in }} \quad \text { and } \quad \mathcal{Q}\left(\frac{I_{\text {in }}}{d_{\text {in }}}\right) \leqslant \frac{I_{\mathcal{H}_{\text {out }}}}{d_{\text {out }}},
$$

where $d_{\text {in }}$ and $d_{\text {out }}$ are the dimensions of the Hilbert spaces $\mathcal{H}_{\text {in }}$ and $\mathcal{H}_{\text {out }}$. We name the maps satisfying Equation (6) timesymmetric quantum operations, and denote the corresponding 
set by $\operatorname{TSOp}\left(\mathcal{H}_{\text {in }}, \mathcal{H}_{\text {out }}\right)$. When the inequalities in Eq. (6) are satisfied with the equality sign, we call the maps timesymmetric quantum channels, and denote the corresponding set by TSChan $\left(\mathcal{H}_{\text {in }}, \mathcal{H}_{\text {out }}\right)$. Note that for $d_{\text {in }}=d_{\text {out }}$, the set of time-symmetric quantum channels coincides with the set of bistochastic channels, and enjoys time symmetry as discussed earlier in Sec. V A.

Like the full set of quantum operations, the set of timesymmetric quantum operations is convex and contains the null operation $\mathcal{Q}=0$. It is rather straightforward to see that the linear map

$$
\Theta: \mathcal{Q} \mapsto \frac{d_{\text {out }}}{d_{\text {in }}} \mathcal{Q}^{\dagger}
$$

is a symmetry transformation from the set $\operatorname{TSOp}\left(\mathcal{H}_{\text {in }}, \mathcal{H}_{\text {out }}\right)$ to the set $\operatorname{TSOp}\left(\mathcal{H}_{\text {out }}, \mathcal{H}_{\text {in }}\right)$. Indeed, it is bijective, it maps convex combinations into convex combinations with the same probabilities, and it transforms null operations into null operations. Likewise, the map

$$
\Theta^{\prime}: \mathcal{Q} \mapsto \frac{d_{\text {out }}}{d_{\text {in }}} \mathcal{Q}^{T}
$$

is also a symmetry transformation from the set $\operatorname{TSOp}\left(\mathcal{H}_{\text {in }}, \mathcal{H}_{\text {out }}\right)$ to the set $\operatorname{TSOp}\left(\mathcal{H}_{\text {out }}, \mathcal{H}_{\text {in }}\right)$.

Both maps $\Theta$ and $\Theta^{\prime}$ transform time-symmetric quantum channels in $\operatorname{TSChan}\left(\mathcal{H}_{\text {in }}, \mathcal{H}_{\text {out }}\right)$ into time-symmetric quantum channels in TSChan $\left(\mathcal{H}_{\text {out }}, \mathcal{H}_{\text {in }}\right)$. For $d_{\text {in }}=d_{\text {out }}$, the maps $\Theta$ and $\Theta^{\prime}$ coincide with the standard time reversal $\mathcal{U} \mapsto U^{\dagger}$ and with the alternative time reversal $\mathcal{U} \mapsto \mathcal{U}^{T}$, respectively.

\section{B. Time-symmetric quantum instruments}

The set of time-symmetric quantum operations can be used to define a time-symmetric variant of quantum theory. In this variant, the allowed experiments are described by time-symmetric quantum instruments, that is, collections of time-symmetric quantum operations $\left(\mathcal{Q}_{n}\right)_{n=1}^{N}$ with the property that the sum $\sum_{n=1}^{N} \mathcal{Q}_{n}$ is a time-symmetric quantum channel, that is, a quantum channel satisfying Eq. (6) with the equality sign. When an experiment is performed on a (possibly subnormalized) state $\rho$, the probability of the outcome $n$ is given by the trace $\operatorname{Tr}\left[\mathcal{Q}_{n}(\rho)\right]$, as in standard quantum theory.

Interestingly, the time-symmetric variant still permits all von Neumann measurements, whose stochastic evolutions are described by quantum operations of the form $\mathcal{Q}_{n}: \rho \mapsto$ $|n\rangle\langle n|\langle n|\rho| n\rangle$, where $(|n\rangle)_{n=1}^{d}$ are the vectors of an orthonormal basis. More generally, the set of time-symmetric quantum instruments contains also the set of Lüders measurements, corresponding to quantum operations of the form $\mathcal{Q}_{n}: \rho \mapsto$ $P_{n} \rho P_{n}$, where $\left(P_{n}\right)_{n=1}^{N}$ is a complete set of orthogonal projectors. In addition, it contains all quantum instruments resulting from sequences of unitary dynamics interspersed with Lüders measurements. These instruments describe the possible closed system evolutions in the framework of consistent histories [58].

The time-symmetric variant also permits all demolition measurements, corresponding to positive operator-valued measures (POVMs) and represented by collections of positive operators $\left(P_{n}\right)_{n=1}^{K}$ satisfying the condition $\sum_{n=1}^{K} P_{n}=I$. Indeed, every quantum operation $\mathcal{Q}_{n}$ defined by the relation
$\mathcal{Q}_{n}(\rho):=\operatorname{Tr}\left[P_{n} \rho\right]$ is a valid time-symmetric operation with one-dimensional output system, according to definition (6).

\section{Relation between standard quantum theory and its time-symmetric variant}

A crucial difference between the time-symmetric variant and standard quantum theory concerns the set of allowed states. In the time-symmetric variant, the only state that can be prepared with unit probability is the maximally mixed state $I / d$. This is because the preparation of a $d$-dimensional quantum system is a quantum operation with one-dimensional input space and $d$-dimensional output space, and setting $d_{\text {in }}=$ 1 and $d_{\text {out }}=d$ in Eq. (6) yields the conditions $\operatorname{Tr}[\rho] \leqslant 1$ and $\rho \leqslant I / d$. In other words, the possible stochastic preparations of the system correspond to subnormalized density matrices satisfying the inequality $\rho \leqslant I / d$. The only normalized state is $\rho=I / d$, while all the other states can be prepared only stochastically, with some probability less than one. For example, a pure quantum state can be prepared with probability at most $1 / d$.

The standard formulation of quantum theory can be retrieved from the time-symmetric variant by introducing an operation of conditioning, whereby probabilistic state preparations are turned into deterministic ones. Mathematically, conditioning amounts to the nonlinear mapping $\rho \mapsto \rho / \operatorname{Tr}[\rho]$. Operationally, it arises from the ability of an agent to observe the outcome of a probabilistic state preparation, and to feed-forward the knowledge of the outcome into future experiments.

Since conditioning enables the deterministic preparation of arbitrary pure states, it also enables the deterministic realization of arbitrary quantum operations. Indeed, every quantum operation $\mathcal{Q}$ can be realized as

$$
\mathcal{Q}(\rho)=\operatorname{Tr}_{\text {aux' }}\left[\left(\mathcal{I}_{\text {out }} \otimes P\right) \mathcal{U}\left(\rho \otimes P_{\psi_{0}}\right)\right],
$$

where $P_{\psi_{0}}$ is a (normalized) pure state of an auxiliary system aux, $\mathcal{U}$ is a joint unitary evolution, transforming states on the Hilbert space $\mathcal{H}_{\text {in }} \otimes \mathcal{H}_{\text {aux }}$ into states on the Hilbert space $\mathcal{H}_{\text {out }} \otimes \mathcal{H}_{\text {aux }}$, for some output auxiliary system aux', and $0 \leqslant P \leqslant I_{\mathrm{aux}^{\prime}}$ is a measurement operator, corresponding to the outcome of a measurement on aux'. Since both the unitary evolution $\mathcal{U}$ and the measurement operator $P$ are allowed in the time-symmetric variant, the addition of all possible states preparations via conditioning yields back the full set of quantum operations in the standard formulation of quantum theory.

\section{Maximality of the time-symmetric variant}

We provided a candidate of time-symmetric variant of quantum theory. A natural question is whether there exist other candidates, and, in the affirmative case, how they would look like. We start by observing that our time-symmetric variant is maximal among all time-symmetric variants of quantum theory in which (1) for every pair of quantum systems $A$ and $B$, the set of allowed operations from system $A$ to system $B$ is a convex subset of the set of quantum operations allowed in quantum theory; (2) for every pair of systems $A$ and $B$, the time reversal of the set of operations from $A$ to $B$ is the set 
of operations from $\bar{B}$ to $\bar{A}$, where $\bar{A}(\bar{B})$ is a suitable quantum system of dimension equal to the dimension of $A(B)$; and (3) every unitary channel is an allowed evolution.

The first two requirements imply that every system has a unique deterministic state.

Lemma 1. In a time-symmetric quantum theory satisfying requirements (1) and (2), the set of subnormalized states of a generic system $B$ contains exactly one deterministic state, that is one density matrix $\omega_{B}$ with $\operatorname{Tr}\left[\omega_{B}\right]=1$.

Proof. Applying requirement 2 in the special case where $A$ is one-dimensional, we obtain that the time reversal of the set of quantum states of system $B$ is the set of measurement operators of some other system $\bar{B}$. By Proposition 1 , this implies that the set of deterministic states of system $B$ coincides with the time reversal of the set of deterministic measurement operators on $\bar{B}$. By requirement 1 , the set of deterministic measurement operators on $\bar{B}$ is a subset of the set of deterministic measurement operators in quantum theory. Since the only deterministic measurement operator in quantum theory is the identity, we conclude that system $B$ has only one deterministic state.

An immediate consequence of the existence of a unique deterministic state is the following characterization of the allowed channels.

Lemma 2. In a time-symmetric quantum theory satisfying requirements (1) and (2), the set of allowed channels with generic input system $A$ and a generic output system $B$ contains only quantum channels $\mathcal{C}$ satisfying the condition $\mathcal{C}\left(\omega_{A}\right)=$ $\omega_{B}$, where $\omega_{A}\left(\omega_{B}\right)$ is the unique deterministic state of system $A(B)$.

The proof is immediate from Lemma 1, which implies that the subset of allowed channels should map the unique deterministic state of system $A$ into the unique deterministic state of system $B$.

The last step of our argument is to use requirement 3, namely that all unitary channels are allowed operations. Combined with Lemma 2, requirement 3 implies that the unique deterministic state of system $A$ should satisfy the condition $\mathcal{U}\left(\omega_{A}\right)=\omega_{A}$ for every unitary channel $\mathcal{U}$ acting on the system. However, the only unitarily invariant state is the maximally mixed state $\omega_{A}=I_{A} / d_{A}$. Hence, Lemma 2 implies that the time-symmetric variant can only contain quantum channels that map maximally mixed states into maximally mixed states. The largest set of such channels is the set of time-symmetric channels defined by Eq. (6).

Summarising, we obtained the following result.

Theorem 5. Every time-symmetric variant of quantum theory satisfying requirements (1)-(3) is contained in the time-symmetric theory defined in Secs. VI A and VIB.

In passing, we note that a generalization of our timesymmetric theory can be obtained by lifting the requirement that all unitary channels are allowed operations. In this case, a time reversal of the allowed quantum operations can be defined in terms of Petz' recovery map [73,75]: for an allowed quantum operation $\mathcal{Q}$ from system $A$ to system $B$, the time reversal is

$$
\Theta(\mathcal{Q}):=\omega_{A}^{1 / 2} \mathcal{Q}^{\dagger}\left(\omega_{B}^{-1 / 2} \rho \omega_{B}^{-1 / 2}\right) \omega_{A}^{1 / 2}
$$

where $\omega_{A}\left(\omega_{B}\right)$ is the unique deterministic state of system $A$ $(B)$, and $\omega_{B}^{-1 / 2}$ denotes the inverse of $\omega_{B}$ on its support.

Alternatively, one could define the time reversal in terms of the transpose map $\mathcal{Q}^{T}$, as

$$
\Theta^{\prime}(\mathcal{Q}):=\omega_{A}^{* 1 / 2} \mathcal{Q}^{T}\left(\omega_{B}^{*-1 / 2} \rho \omega_{B}^{*-1 / 2}\right) \omega_{A}^{* 1 / 2},
$$

where $\omega_{A}^{*}$ and $\omega_{B}^{*}$ are the complex conjugates of $\omega_{A}$ and $\omega_{B}$, respectively.

The time-reversal symmetries considered earlier in Eqs. (7) and (8) are a special case of the symmetries (10) and (11), corresponding to the choice of maximally mixed states $\omega_{A}=$ $I_{\text {in }} /$ in and $\omega_{B}=I_{\text {out }} / d_{\text {out }}$ as the unique deterministic states of systems $A$ and $B$.

\section{RELAXATIONS OF THE NOTION OF SYMMETRY}

Here we briefly discuss two relaxations of the notion of operation symmetry, and we show that the set of all quantum operations admits a time symmetry in these two relaxed senses.

\section{A. Nonbijective symmetries}

One way to evade Theorem 4 is to relax the idea that a symmetry should be bijective. In particular, for finite dimensional systems one could consider the transformation $\mathcal{S}: \operatorname{Op}\left(\mathcal{H}_{\text {in }}, \mathcal{H}_{\text {out }}\right) \rightarrow \operatorname{Op}\left(\mathcal{H}_{\text {out }}, \mathcal{H}_{\text {in }}\right), \mathcal{Q} \mapsto \mathcal{Q}^{\dagger} / d_{\text {in }}$. This transformation $\mathcal{S}$ is maps quantum operations into quantum operations, is compatible with randomization, and maps the null operation into the null operation. It is injective, meaning that it maps distinct quantum operations into distinct quantum operations. However, it falls short of being surjective: there exist quantum operations that are not of the form $\mathcal{S}(\mathcal{Q})$ for any quantum operation $\mathcal{Q}$. If the map $\mathcal{S}$ were used to describe time reversal, then the set of evolutions observed by an agent with inverted time arrow would be strictly smaller than the set of all quantum evolutions. Basically, the time-reversed agent would only observe a scaled down version of the set of all possible quantum evolutions. Adopting the standard interpretation that the trace of the output state corresponds to the probability that a transformation occurs, this would mean that a time reversed transformation $\mathcal{S}(\mathcal{Q})$ would take place with probability at most $1 / d_{\text {in }}$.

Note that the symmetry transformation $\mathcal{S}$ is probabilistically reversible: if applied twice, it returns the original quantum operation $\mathcal{Q}$, scaled down by a factor $1 / d_{\text {in }}^{2}$. If this factor is ignored, one may say that the set of quantum operations enjoys a symmetry under time reversal. We call such symmetries weak symmetries, to stress that they only hold up to an overall scaling factor. Most of the existing proposals of time-symmetric formulations of quantum theory [48-50,52] exhibit this type of weak symmetry. Physically, the relevance of weak symmetry is argued based on post-selection: if an agent postselects on the occurrence of a quantum operation, then any scaling factor becomes irrelevant.

\section{B. Nonlinear symmetries}

In this paper, we defined operation symmetries as transformations that preserve the convex structure and the notion 
of zero probability, by mapping the null operation into itself. These two requirements imply that every symmetry transformation acts linearly on the vector space spanned by quantum operations (see Proposition 2 in Appendix D).

A way to evade Theorem 4 is to give up the above requirements and to consider nonlinear symmetries, such as those considered in nonlinear modifications of quantum mechanics [82-84]. These modifications, however, generally come with a significant change of the operational content of quantum theory. For example, the state space in nonlinear quantum mechanics is generally different from the space of density matrices, and ensembles of pure states that are indistinguishable in ordinary quantum mechanics may become distinguishable in nonlinear modifications [85].

We now show that, if one gives up the requirement of linearity, a time reversal can be defined for all possible quantum operations. A nonlinear time reversal was introduced by Crooks in the case of quantum channels with $\mathcal{H}_{\text {in }}=\mathcal{H}_{\text {out }}$ [86]. Crooks' definition of time reversal coincides with Petz' recovery map [73,75]: given a quantum channel $\mathcal{C}$ and a quantum state $\rho_{0}$ such that $\mathcal{C}\left(\rho_{0}\right)=\rho_{0}$, Crooks' time reversal is the recovery map $\mathcal{C}_{\rho_{0}}$ defined as $\mathcal{C}_{\rho_{0}}(\rho):=$ $\rho_{0}^{1 / 2} \mathcal{C}^{\dagger}\left(\rho_{0}^{-1 / 2} \rho \rho_{0}^{-1 / 2}\right) \rho_{0}^{1 / 2}$, where $\rho_{0}^{-1}$ is inverse of $\rho_{0}$ on its support. For bistochastic channels, one can choose $\rho_{0}$ to be the maximally mixed state, and this definition of time reversal coincides with the definition in Eq. (7). In general, the map $\Theta: \mathcal{C} \mapsto \mathcal{C}_{\rho_{0}}$ is nonlinear, because it depends on the choice of a fixed point $\rho_{0}$ for the channel $\mathcal{C}$.

More generally, Crooks' definition can be extended to channels with different input and output spaces, by fixing an arbitrary state $\rho_{0} \in \operatorname{St}\left(\mathcal{H}_{\text {in }}\right)$ and defining the state-dependent time reversal $\mathcal{C}_{\rho_{0}}$ as

$$
\mathcal{C}_{\rho_{0}}(\rho):=\rho_{0}^{1 / 2} \mathcal{C}^{\dagger}\left(\left[\mathcal{C}\left(\rho_{0}\right)\right]^{-1 / 2} \rho\left[\mathcal{C}\left(\rho_{0}\right)\right]^{-1 / 2}\right) \rho_{0}^{1 / 2} .
$$

Note, however, that the above definition is noncanonical, due to the arbitrariness in the choice of the state $\rho_{0}$.

Similarly, a time reversal could be defined for arbitrary quantum operations. Given a quantum operation $\mathcal{Q}$, one could (1) fix a complementary quantum operation $\mathcal{Q}^{\prime}$ such that the map $\mathcal{C}_{0}:=\mathcal{Q}+\mathcal{Q}^{\prime}$ is a quantum channel, and (2) fix an arbitrary quantum state $\rho_{0} \in \operatorname{St}\left(\mathcal{H}_{\text {in }}\right)$. Then, a time reversal $\mathcal{Q}_{\mathcal{C}_{0}, \rho_{0}}$ could be defined via the relation

$$
\mathcal{Q}_{\mathcal{C}_{0}, \rho_{0}}(\rho):=\rho_{0}^{1 / 2} \mathcal{Q}^{\dagger}\left(\left[\mathcal{C}\left(\rho_{0}\right)\right]^{-1 / 2} \rho\left[\mathcal{C}\left(\rho_{0}\right)\right]^{-1 / 2}\right) \rho_{0}^{1 / 2} .
$$

This definition would guarantee that $\mathcal{Q}_{\mathcal{C}_{0}, \rho_{0}}$ is a valid quantum operation. Still, the map $\Theta: \mathcal{Q} \mapsto \mathcal{Q}_{\mathcal{C}_{0}, \rho_{0}}$ is nonlinear and noncanonical, due to the arbitrariness in the choice of channel $\mathcal{C}_{0}$ and state $\rho_{0}$.

\section{CONCLUSIONS}

In this work we introduced a notion of symmetry for quantum evolutions, defined as a one-to-one transformation that is consistent with randomizations and with the notion of zero probability. We established a Wigner theorem for quantum operations, showing that every symmetry of quantum operations can be broken down into two symmetries of quantum states, which are either both unitary or both antiunitary.

We then showed that the time-reversal symmetry of unitary quantum dynamics cannot be extended to a symmetry of the full set of stochastic quantum evolutions. In other words, the set of all quantum evolutions is incompatible with time symmetry. Our no-go theorem implies that it is impossible to translate the quantum description of experiments made by an ordinary agent into a quantum description of experiments made by a hypothetical agent with time-reversed arrow of time: if the ordinary agent can perform all possible quantum experiments (and therefore all possible quantum operations), then its experiments cannot be described by the time-reversed agent.

It is worth noting that the impossibility of defining a time-reversal symmetry is not necessarily unique to quantum theory. As a matter of fact, most of the steps in the proof of our no-go result can be reproduced also in classical probability theory, leading to a no-go theorem for time-reversal symmetries of classical stochastic evolutions. What makes the quantum case more interesting, however, is that in the classical world there is a natural notion of time-symmetric theory, namely deterministic classical theory, with only pure states and reversible classical evolutions. In quantum theory, instead, defining a time-symmetric theory is more problematic, at least in the standard operational interpretation where measurements play a central role. One could, of course, consider the fragment of quantum theory containing only pure states and unitary evolutions, as it is done in Everett's interpretation of quantum mechanics. If this route is taken, the remaining difficulty would be to give a consistent account of how the theory is to be used to make predictions about the outcomes of experiments [87]. On the other hand, if even just a single projective measurement is added on top of pure states and unitary evolutions, then all quantum operations can be generated, and time symmetry is broken.

After establishing our no-go theorem for time symmetry in the standard framework, we formulated a time-symmetric variant of quantum theory in which the deterministic operations are required to preserve the maximally mided state. We showed that our time-symmetric variant is maximal among all time-symmetric variants where all unitary channels are allowed evolutions, and that the standard operational framework of quantum theory can be retrieved from the time-symmetric variant through an operation of conditioning on the outcomes of state preparations.

The results of this work provide a rigorous framework for the analysis of dynamical symmetries in quantum theory. We hope that the framework and the analysis initiated in this work will inform future developments in the foundations of quantum mechanics and in quantum information theory.

\section{ACKNOWLEDGMENTS}

It is a pleasure to thank Ingemar Bengtsson, Vern Paulsen, Mizanur Rahaman, and Erling Størmer for a fruitful correspondence. GC acknowledges A Winter for an insightful discussion on Petz' recovery map that led to Eqs. (7), (8), and (12), and to $\mathrm{J}$ Barrett for helpful comments on an earlier version of the results. This work was supported by the National Natural Science Foundation of China through Grant No. 11675136, by the Hong Kong Research Grant Council through Grant No. 17307719 and through the Senior Research Fellowship Scheme SRFS2021-7S02, by the Croucher 
Foundation, by the John Templeton Foundation through Grant No. 61466, The Quantum Information Structure of Spacetime (qiss.fr), by the Swedish Research Council through Grant No. 2020-04980, by the Foundation for Polish Science through TEAM-NET project (contract No. POIR.04.04.0000-17C1/18-00), and by the Polish National Science Centre under the project number DEC-2015/18/A/ST2/00274. Research at the Perimeter Institute is supported by the Government of Canada through the Department of Innovation, Science and Economic Development Canada and by the Province of Ontario through the Ministry of Research, Innovation and Science. The opinions expressed in this publication are those of the authors and do not necessarily reflect the views of the John Templeton Foundation.

\section{APPENDIX A: PROOF OF THEOREM 2}

The proof of Theorem 2 is based on the following arguments. (1) Every state space symmetry must map pure states into pure states, and therefore it induces a transformation of ray space, (2) the induced transformation of ray space preserves the ray product, (3) hence, every state space symmetry induces a ray space symmetry, which can be characterized using (the original formulation of) Wigner's theorem.

In the following, we provide the proof of all the steps used in this argument. Let us start by showing that every state space symmetry maps pure states into pure states. For a given vector $\psi \in \mathcal{H}$, we will use the notation $P_{\psi}:=|\psi\rangle\langle\psi|$.

Lemma 3. Every state space symmetry $\mathcal{S}$ maps pure states into pure states. The correspondence $P_{\psi} \mapsto \mathcal{S}\left(P_{\psi}\right)$ is bijective.

Proof. The proof is standard and is reproduced here just for completeness. For a generic unit vector $\psi \in \mathcal{K}$, the operator $\mathcal{S}\left(P_{\psi}\right)$ is a density matrix. Hence, $\mathcal{S}\left(P_{\psi}\right)$ can be decomposed as as $\mathcal{S}\left(P_{\psi}\right)=\sum_{i} p_{i} P_{\psi_{i}}$ where $\left\{p_{i}\right\}$ are probabilities and $\left\{\left|\psi_{i}\right\rangle\right\} \in \mathcal{H}$ are unit vectors. Since $S$ is a state space symmetry, one has $P_{\psi}=\mathcal{S}^{-1} \circ \mathcal{S}\left(P_{\psi}\right)=\sum_{i} p_{i} \mathcal{S}^{-1}\left(P_{\psi_{i}}\right)$, and, therefore $\mathcal{S}^{-1}\left(P_{\psi_{i}}\right)=P_{\psi}$ for all $i$. Applying $\mathcal{S}$ on both sides of the equality, one obtains $P_{\psi_{i}}=\mathcal{S}\left(P_{\psi}\right), \forall i$. Hence, $\mathcal{S}\left(P_{\psi}\right)$ is a pure state.

The correspondence $P_{\psi} \mapsto \mathcal{S}\left(P_{\psi}\right)$ is injective, because $\mathcal{S}$ is injective on the whole space of density matrices. Moreover, $\mathcal{S}$ is surjective: since the inverse transformation $\mathcal{S}^{-1}$ maps pure states into pure states, every pure state $P_{\phi}$ is the image of the pure state $\mathcal{S}^{-1}\left(P_{\phi}\right)$ under the map $\mathcal{S}$.

Now, recall that the set of rank-one projectors is in oneto-one correspondence with the set of unit rays. For every rank-one projector $P$, let ray $(P)$ be the corresponding unit ray, defined as

$$
\operatorname{ray}(P):=\left\{\frac{P \psi}{\|P \psi\|} \mid \psi \in \mathcal{H}, P \psi \neq 0\right\} .
$$

Owing to the one-to-one correspondence between rank-one projectors and unit rays, we have the following.

Corollary 1 . Every state space symmetry $\mathcal{S}: \operatorname{St}(\mathcal{H}) \rightarrow$ $\operatorname{St}(\mathcal{K})$ induces a bijective transformation $\underline{S}: \underline{\mathcal{H}} \rightarrow \underline{\mathcal{K}}$ via the relation

$$
\underline{S}(\underline{\psi}):=\operatorname{ray}\left(\mathcal{S}\left(P_{\psi}\right)\right) .
$$

The next step is to show that the ray space transformation $\underline{S}$ preserves the ray product. In preparation for this, we introduce some notation. For two generic Hilbert-Schmidt operators $X$ : $\mathcal{H} \rightarrow \mathcal{H}$ and $Y: \mathcal{H} \rightarrow \mathcal{H}$, we denote their Hilbert-Schmidt product by

$$
\langle X, Y\rangle:=\operatorname{Tr}\left[X^{\dagger} Y\right] .
$$

The ray product of two unit rays $\psi$ and $\phi$ can be expressed in terms of the Hilbert-Schmidt product as

$$
\underline{\psi} \cdot \underline{\phi}=\sqrt{\left\langle P_{\psi}, P_{\phi}\right\rangle}
$$

For rank-one projectors $P_{\psi}$ and $P_{\phi}$, the Hilbert-Schmidt product can be equivalently expressed as

$$
\left\langle P_{\psi}, P_{\phi}\right\rangle=F\left(P_{\psi}, P_{\phi}\right),
$$

where $F(\rho, \sigma):=(\operatorname{Tr}[\sqrt{\sqrt{\rho} \sigma \sqrt{\rho}}])^{2}$ is the (square of the) Uhlmann fidelity $[88,89]$, defined for any two density matrices $\rho$ and $\sigma$.

The last definition we need is the definition of state space homomorphism.

Definition 4. A map $\mathcal{J}: \operatorname{St}(\mathcal{H}) \rightarrow \operatorname{St}(\mathcal{K})$ is a state space homomorphism if it preserves convex combinations.

Recall that every state space homomorphism can be equivalently represented in the "Heisenberg picture," as a map on observables. Specifically, the Heisenberg picture of the map $\mathcal{J}: \operatorname{St}(\mathcal{H}) \rightarrow \operatorname{St}(\mathcal{K})$ is provided by the dual map $\mathcal{J}^{\dagger}:$ $L(\mathcal{K}) \rightarrow L(\mathcal{H})$, uniquely defined by the condition

$$
\left\langle\mathcal{J}^{\dagger}(A), P_{\psi}\right\rangle=\left\langle A, \mathcal{J}\left(P_{\psi}\right)\right\rangle \quad \forall A \in B(\mathcal{K}), \forall \psi \in \mathcal{H} .
$$

Lemma 4. A map $\mathcal{J}: \operatorname{St}(\mathcal{H}) \rightarrow \operatorname{St}(\mathcal{K})$ is a state space homomorphism if and only if the dual map $\mathcal{J}^{\dagger}: B(\mathcal{K}) \rightarrow B(\mathcal{H})$ is positive and unital.

Proof. We recall that a map is positive if it maps nonnegative operators into non-negative operators. Let $P: \mathcal{K} \rightarrow$ $\mathcal{K}$ be a non-negative operator. Then, for every unit vector $\psi \in$ $\mathcal{H}$, one has $\left\langle\psi\left|\mathcal{J}^{\dagger}(P)\right| \psi\right\rangle=\left\langle\mathcal{J}^{\dagger}(P), P_{\psi}\right\rangle=\left\langle P, \mathcal{J}\left(P_{\psi}\right)\right\rangle=$ $\operatorname{Tr}\left[P \mathcal{J}\left(P_{\psi}\right)\right] \geqslant 0$, the last inequality following from the fact that $\mathcal{J}\left(P_{\psi}\right)$ is a density matrix. Hence, $\mathcal{J}^{\dagger}(P)$ is a nonnegative operator, and $\mathcal{J}^{\dagger}$ is positive.

Similarly, it is easy to see that $\mathcal{J}^{\dagger}$ is unital, namely $\mathcal{J}^{\dagger}\left(I_{\mathcal{K}}\right)=I_{\mathcal{H}}$. Indeed, for every unit vector $\psi \in \mathcal{H}$, one has $\left\langle\psi\left|\mathcal{J}^{\dagger}\left(I_{\mathcal{K}}\right)\right| \psi\right\rangle=\left\langle\mathcal{J}^{\dagger}\left(I_{\mathcal{K}}\right), P_{\psi}\right\rangle=\left\langle I_{\mathcal{K}}, \mathcal{J}\left(P_{\psi}\right)\right\rangle=$ $\operatorname{Tr}\left[\mathcal{J}\left(P_{\psi}\right)\right]=1$, because $\mathcal{J}\left(P_{\psi}\right)$ is a density matrix. Summarising, the map $\mathcal{J}^{\dagger}$ is positive and unital.

Conversely, if the map $\mathcal{J}^{\dagger}$ is positive and unital, then the map $\mathcal{J}$ transforms density matrices into density matrices: indeed, it transforms positive operators into positive operators and unit-trace operators into unit-trace operators, as one can see by running the above arguments in reverse. Moreover, since $\mathcal{J}^{\dagger}$ is linear, the map $\mathcal{J}=\left(\mathcal{J}^{\dagger}\right)^{\dagger}$ is linear, too. In particular, it is convex-linear. Hence, $\mathcal{J}$ induces a map from $\operatorname{St}(\mathcal{H})$ to $\operatorname{St}(\mathcal{K})$ that is compatible with convex combinations.

An important property of state space homomorphisms is that they cannot increase the distinguishability of quantum states, or equivalently, they cannot decrease the similarity of quantum states. Taking the fidelity as the measure of similarity, we have the following lemma.

Lemma 5. Every state space homomorphism $\mathcal{J}$ : $\operatorname{St}(\mathcal{H}) \rightarrow \operatorname{St}(\mathcal{K})$ is fidelity nondecreasing, namely,

$$
F(\mathcal{J}(\rho), \mathcal{J}(\sigma)) \geqslant F(\rho, \sigma) \quad \forall \rho, \sigma \in \operatorname{St}(\mathcal{K}) .
$$

If $\mathcal{J}$ is a state space symmetry, then the equality holds. 
Proof. The fidelity between two generic density matrices $\rho$ and $\sigma$ can be equivalently expressed as

$$
F(\rho, \sigma)=\left(\max _{\left\{P_{i}\right\}} \sum_{i} \sqrt{\operatorname{Tr}\left[P_{i} \rho\right] \operatorname{Tr}\left[P_{i} \sigma\right]}\right)^{2},
$$

where the maximum is over all positive operator-valued measures (POVMs) $\left(P_{i}\right)$, consisting of positive semidefinite operators $P_{i} \geqslant 0$ satisfying the normalization condition $\sum_{i} P_{i}=I$. Hence, one has

$$
\begin{aligned}
F & (\mathcal{J}(\rho), \mathcal{J}(\sigma)) \\
& =\left(\max _{\left\{P_{i}\right\}} \sum_{i} \sqrt{\operatorname{Tr}\left[\mathcal{J}^{\dagger}\left(P_{i}\right) \rho\right] \operatorname{Tr}\left[\mathcal{J}^{\dagger}\left(P_{i}\right) \sigma\right]}\right)^{2} \\
& \geqslant\left(\max _{\left\{Q_{i}\right\}} \sum_{i} \sqrt{\operatorname{Tr}\left[Q_{i} \rho\right] \operatorname{Tr}\left[Q_{i} \sigma\right]}\right)^{2} \\
& =F(\rho, \sigma)
\end{aligned}
$$

where the inequality follows from the fact that the operators $Q_{i}:=\mathcal{J}^{\dagger}\left(P_{i}\right)$ form a POVM, because $\mathcal{J}^{\dagger}$ is positive and unital (Lemma 4).

If $\mathcal{J}$ is a state space symmetry, then one has $F(\rho, \sigma)=$ $F\left(\mathcal{J}^{-1} \circ \mathcal{J}(\rho), \mathcal{J}^{-1} \circ \mathcal{J}(\sigma)\right) \geqslant F(\mathcal{J}(\rho), \mathcal{J}(\sigma)) \geqslant F(\rho, \sigma)$, whence the equality $F(\mathcal{J}(\rho), \mathcal{J}(\sigma))=F(\rho, \sigma)$.

We are now ready to show that every state space symmetry induces a ray space symmetry.

Lemma 6. For every state space symmetry $\mathcal{S}: \operatorname{St}(\mathcal{H}) \rightarrow$ $\operatorname{St}(\mathcal{K})$, the associated ray space transformation $\underline{S}: \underline{\mathcal{H}} \rightarrow \underline{\mathcal{K}}$, defined in Eq. (A2), preserves the ray product.

Proof. For two generic unit vectors $\psi$ and $\phi$, Eqs. (A4) and (A5) imply $\psi \cdot \phi=\sqrt{\left\langle P_{\psi}, P_{\phi}\right\rangle}=\sqrt{F\left(P_{\psi}, P_{\phi}\right)}$. On the other hand, Lemma 5 guarantees the equality $F\left(P_{\psi}, P_{\phi}\right)=F\left(\mathcal{S}\left(P_{\psi}\right), \mathcal{S}\left(P_{\phi}\right)\right)$. In turn, Eqs. (A5) and (A4) imply $F\left(\mathcal{S}\left(P_{\psi}\right), \mathcal{S}\left(P_{\phi}\right)\right)=\left\langle\mathcal{S}\left(P_{\psi}\right), \mathcal{S}\left(P_{\phi}\right)\right\rangle=$ $\left[\operatorname{ray}\left(\mathcal{S}\left(P_{\psi}\right)\right) \cdot \operatorname{ray}\left(\mathcal{S}\left(P_{\phi}\right)\right)\right]^{2}$, with the notation $\operatorname{ray}(\cdot)$ defined as in Eq. (A1). Finally, the definition of the map $\underline{S}$ in Eq. (A2) yields the equality $\operatorname{ray}\left(\mathcal{S}\left(P_{\psi}\right)\right) \cdot \operatorname{ray}\left(\mathcal{S}\left(P_{\phi}\right)\right)=\underline{S}(\psi) \cdot \underline{S}(\phi)$. Hence, we obtained the equality $\psi \cdot \phi=\underline{S}(\psi) \cdot \underline{S}(\bar{\phi})$. Since the unit vectors $\psi$ and $\phi$ are arbitrary, we conclude that $\underline{S}$ preserves the ray product.

We then conclude with the proof of Theorem 2.

Proof of Theorem 2. For a given space symmetry $\mathcal{S}$ : $\operatorname{St}(\mathcal{H}) \rightarrow \operatorname{St}(\mathcal{K})$, the corresponding ray space transformation $\underline{S}: \mathcal{H} \rightarrow \mathcal{K}$ is bijective (corollary 1 ) and preserves the ray product (Lemma 6 ). Hence, it is a ray space symmetry.

By Wigner's theorem, $\underline{S}$ has either the form $\underline{S}(\psi)=U \psi$, or the form $\underline{S}(\psi)=U \psi^{*}$, for some unitary operator $\bar{U}: \overline{\mathcal{H} \rightarrow}$ $\mathcal{K}$. In terms of rank-one projectors, this means that one has either $\mathcal{S}\left(P_{\psi}\right)=U P_{\psi} U^{\dagger}$ or $\mathcal{S}\left(P_{\psi}\right)=U P_{\psi^{*}} U^{\dagger}=U P_{\psi}^{T} U^{\dagger}$. Since the map $\mathcal{S}$ is uniquely determined by its action on the pure states, the action of $\mathcal{S}$ on a generic density matrix $\rho$ is either of the form $\mathcal{S}(\rho)=U \rho U^{\dagger}$ or of the form $\mathcal{S}(\rho)=U \rho^{T} U^{\dagger}$.

\section{APPENDIX B: EQUIVALENCE BETWEEN THE ORIGINAL WIGNER THEOREM AND ITS DENSITY MATRIX FORMULATION}

In the previous Appendix, we have seen that the original formulation of Wigner's theorem (Theorem 1) implies the characterization of state space symmetries of Theorem 2 . We now show that the converse also holds: Theorem 2 implies Wigner's theorem.

Let $\underline{S}: \underline{\mathcal{H}} \rightarrow \underline{\mathcal{K}}$ be a ray space symmetry. Our goal is to show that $\underline{S}$ is induced by a unitary or antiunitary transformation in Hilbert space. Note that there is a one-to-one correspondence between the unit vectors $\psi \otimes \psi^{*} \in \mathcal{H} \otimes \mathcal{H}$ and the unit rays $\psi \in \underline{\mathcal{H}}$. Hence, the transformation $\underline{S}$ induces a transformation of the vectors $\psi \otimes \psi^{*}$, hereafter denoted by $S_{\text {prod. If }} \underline{S}(\psi)=\psi^{\prime}$, then $S_{\text {prod }}\left(\psi \otimes \psi^{*}\right)=\psi^{\prime} \otimes \psi^{\prime *}$.

Now, let $\left\{\psi_{i}\right\} \overline{\mathcal{H}}$ be a set of unit vectors with the property that $\left\{\psi_{i} \otimes \psi_{i}^{*}\right\}$ is a spanning set for the space $\mathcal{H} \otimes \mathcal{H}$. Then, we have

$$
\begin{aligned}
& \left\langle S_{\text {prod }}\left(\psi_{i} \otimes \psi_{i}^{*}\right) \mid S_{\text {prod }}\left(\psi_{j} \otimes \psi_{j}^{*}\right)\right\rangle=\left\langle\psi_{i}^{\prime} \otimes \psi_{i}^{\prime *} \mid \psi_{j}^{\prime} \otimes \psi_{j}^{\prime *}\right\rangle \\
& =\left|\left\langle\psi_{i}^{\prime} \mid \psi_{j}^{\prime}\right\rangle\right|^{2} \\
& =\underline{\psi_{i}^{\prime}} \cdot \underline{\psi_{j}^{\prime}} \\
& =\underline{\psi_{i}} \cdot \underline{\psi_{j}} \\
& =\left|\left\langle\psi_{i} \mid \psi_{j}\right\rangle\right|^{2} \\
& =\left\langle\psi_{i} \otimes \psi_{i}^{*} \mid \psi_{j} \otimes \psi_{j}^{*}\right\rangle \text {. }
\end{aligned}
$$

In other words, the two sets $\left\{\psi_{i} \otimes \psi_{i}^{*}\right\}$ and $\left\{\psi_{i}^{\prime} \otimes \psi_{i}^{\prime *}\right\}$ have the same Gram matrix. This condition guarantees that there exists a unitary transformation $W: \mathcal{H} \otimes \mathcal{H} \rightarrow \mathcal{K} \otimes \mathcal{K}$ such that $\psi_{i}^{\prime} \otimes \psi_{i}^{\prime *}=W\left(\psi_{i} \otimes \psi_{i}^{*}\right)$ (for a proof, see, e.g., property 3 of Ref. [90]). The transformation $W$ is uniquely defined, because the states $\left\{\psi_{i} \otimes \psi_{i}^{*}\right\}$ are a basis. Using this fact, we can guarantee the equality $\psi^{\prime} \otimes \psi^{\prime *}=W\left(\psi \otimes \psi^{*}\right)$ for every unit vector $\psi$. Hence, the transformation $\mathcal{S}_{\text {prod }}$ is linear.

Now, since the product vectors $\psi \otimes \psi^{*}$ are in one-to-one linear correspondence with the rank-one projectors $P_{\psi}$, the linear transformation $S_{\text {prod }}$ induces a linear transformation $\mathcal{S}$ on the rank-one projectors $P_{\psi}$. By linearity, the transformation $\mathcal{S}$ acts on the set of density matrices, and is consistent with randomizations. Moreover, since $S_{\text {prod }}$ is a bijection, also $\mathcal{S}$ is. Hence, it is a symmetry of the set of quantum states. By applying Theorem 2, we then obtain that $\mathcal{S}$ is either a unitary transformation $\mathcal{S}: \rho \mapsto U \rho U^{\dagger}$ or an antiunitary transformation $\mathcal{S}: \rho \mapsto U \rho^{T} U^{\dagger}$. Restricting its action on pure states, we have that the pure state $|\psi\rangle\langle\psi|$ is transformed either into $U|\psi\rangle\langle\psi| U^{\dagger}$ or into $U(|\psi\rangle\langle\psi|)^{T} U^{\dagger}=U\left|\psi^{*}\right\rangle\left\langle\psi^{*}\right| U^{\dagger}$. Translating back in terms of the map $\underline{S}$, we obtain that $\underline{S}(\psi)$ is either equal to $U \psi$ or equal to $U \psi^{*}$. Hence, $\underline{S}$ is either induced by a unitary transformation or by an antiunitary transformation. This concludes the derivation of Wigner's theorem from Theorem 2.

\section{APPENDIX C: PROOF OF PROPOSITION 1}

Here we prove that every symmetry of quantum operations maps channels into channels. 
Let $\mathcal{S}: \operatorname{Op}\left(\mathcal{H}_{\text {in }}, \mathcal{H}_{\text {out }}\right) \rightarrow \operatorname{Op}\left(\mathcal{K}_{\text {in }}, \mathcal{K}_{\text {out }}\right)$ be a symmetry of quantum operations, and let $\mathcal{C} \in \operatorname{Chan}\left(\mathcal{H}_{\text {in }}, \mathcal{H}_{\text {out }}\right)$ be a quantum channel. Since the set of quantum channels is a subset of the set of quantum operations, $\mathcal{S}(\mathcal{C})$ is a quantum operation.

Now, for every quantum operation $\mathcal{Q}$ there exists another quantum operation $\mathcal{Q}^{\prime}$ such that $\mathcal{Q}+\mathcal{Q}^{\prime}$ is a quantum channel. Let $\mathcal{Q}^{\prime}$ be the quantum operation such that $\mathcal{C}^{\prime}:=$ $\mathcal{S}(\mathcal{C})+\mathcal{Q}^{\prime}$ is a quantum channel. Applying the inverse map $\mathcal{S}^{-1}$ to both sides of the equation, we obtain $\mathcal{S}^{-1}\left(\mathcal{C}^{\prime}\right)=$ $\mathcal{C}+\mathcal{S}^{-1}\left(\mathcal{Q}^{\prime}\right)$. Since the map $\mathcal{S}^{-1}$ transforms quantum operations into quantum operations, the map $\mathcal{C}+\mathcal{S}^{-1}\left(\mathcal{Q}^{\prime}\right)$ must be trace nonincreasing. Since both terms in the sum are completely positive, and since $\mathcal{C}$ is trace preserving, we obtain the condition $\mathcal{S}^{-1}\left(\mathcal{Q}^{\prime}\right)=0$. Hence, we obtained $\mathcal{S}^{-1}\left(\mathcal{C}^{\prime}\right)=$ $\mathcal{C}$. Applying the map $\mathcal{S}$ on both sides, we finally conclude $\mathcal{C}^{\prime}=\mathcal{S}(\mathcal{C})$, that is, $\mathcal{S}(\mathcal{C})$ is a quantum channel. Since $\mathcal{C}$ was a generic channel, we conclude that $\mathcal{S}$ maps channels into channels.

\section{APPENDIX D: PROOF OF THEOREM 3}

In this section we first review the technical tools used in the proof, and then provide the derivation of Wigner's theorem for quantum operations.

\section{a. Supermaps}

First of all, we observe that every symmetry of quantum operations induces a linear map acting on the vector space spanned by quantum operations. Such higher-order linear maps are called supermaps [8-11,13], and can be used for a variety of purposes. The special type of supermaps that transform quantum operations into quantum operations were characterized in Ref. [8] in the finite dimensional case, and in Ref. [11] in the infinite dimensional case. Another type of supermaps was defined in Ref. [13], which considered transformations of a certain convex set of completely positive maps into itself, and used them to define the dynamics in an extended version of quantum theory where the state space of a $d$-dimensional system has dimension $d^{4}$.

To avoid technical complications, in the following we will restrict ourselves to the finite-dimensional case. In the following, we will use the notation $L(\mathcal{H}, \mathcal{K})$ for the space of linear operators on a generic Hilbert space $\mathcal{H}$ to a generic Hilbert space $\mathcal{K}$, the notation $L(\mathcal{H}):=L(\mathcal{H}, \mathcal{H})$ for the linear operators on $\mathcal{H}$, and the notation $\operatorname{Map}(\mathcal{H}, \mathcal{K})$ for the set of linear maps from $L(\mathcal{H})$ to $L(\mathcal{K})$.

Proposition 2. Every map $\mathcal{S}: \operatorname{Op}\left(\mathcal{H}_{\text {in }}, \mathcal{H}_{\text {out }}\right) \rightarrow$ $\mathrm{Op}\left(\mathcal{K}_{\text {in }}, \mathcal{K}_{\text {out }}\right)$ that is consistent with randomizations and preserves the null operation can be uniquely extended to a linear map $\mathcal{S}_{\text {lin }}: \operatorname{Map}\left(\mathcal{H}_{\text {in }}, \mathcal{H}_{\text {out }}\right) \rightarrow \operatorname{Map}\left(\mathcal{K}_{\text {in }}, \mathcal{K}_{\text {out }}\right)$.

Proof. Since the map $\mathcal{S}$ is consistent with randomizations and preserves the null operation, we have

$$
\begin{aligned}
\mathcal{S}(p \mathcal{Q}) & =\mathcal{S}(p \mathcal{Q}+(1-p) 0) \\
& =p \mathcal{S}(\mathcal{Q})+(1-p) \mathcal{S}(0) \\
& =p \mathcal{S}(\mathcal{Q}),
\end{aligned}
$$

for every probability $p \in[0,1]$ and for every quantum operation $\mathcal{Q}$.
Due to Eq. (D1), any two quantum operations $\mathcal{Q}$ and $\mathcal{Q}^{\prime}$ satisfying the condition $\lambda^{\prime} \mathcal{Q}^{\prime}=\lambda \mathcal{Q}$ for positive numbers $\lambda$ and $\lambda^{\prime}$, must also satisfy the condition $\lambda^{\prime} \mathcal{S}\left(\mathcal{Q}^{\prime}\right)=\lambda \mathcal{S}(\mathcal{Q})$.

Now, we show that the map $\mathcal{S}$ is linear on conic combinations: if $\sum_{i} \lambda_{i} \mathcal{Q}_{i}=\sum_{j} \lambda_{j}^{\prime} \mathcal{Q}_{j}^{\prime}$, then

$$
\sum_{i} \lambda_{i} \mathcal{S}\left(\mathcal{Q}_{i}\right)=\sum_{j} \lambda_{j}^{\prime} \mathcal{S}\left(\mathcal{Q}_{j}^{\prime}\right)
$$

for every set of quantum operations $\left\{\mathcal{Q}_{i}\right\}\left(\left\{\mathcal{Q}_{j}^{\prime}\right\}\right)$ and positive coefficients $\left\{\lambda_{i}\right\}\left(\left\{\lambda_{j}^{\prime}\right\}\right)$.

To prove Eq. (D2), we define the probability distributions $\left(p_{i}\right)$ and $\left(p_{j}\right)$ as

$$
p_{i}:=\frac{\lambda_{i}}{\lambda}, \quad \lambda:=\sum_{i} \lambda_{i}, \quad p_{j}^{\prime}:=\frac{\lambda_{j}^{\prime \prime}}{\lambda}, \quad \lambda^{\prime}:=\sum_{j} \lambda_{j}^{\prime},
$$

and the quantum operations

$$
\mathcal{Q}:=\sum_{i} p_{i} \mathcal{Q}_{i}, \quad \mathcal{Q}^{\prime}:=\sum_{j} p_{j}^{\prime} \mathcal{Q}_{j}^{\prime}
$$

With these definitions we have the equality $\lambda^{\prime} \mathcal{Q}^{\prime}=\lambda \mathcal{Q}$, which implies $\lambda^{\prime} \mathcal{S}\left(\mathcal{Q}^{\prime}\right)=\lambda \mathcal{S}(\mathcal{Q})$. Hence, we have

$$
\begin{aligned}
\sum_{i} \lambda_{i} \mathcal{S}\left(\mathcal{Q}_{i}\right) & =\lambda \sum_{i} p_{i} \mathcal{S}\left(\mathcal{Q}_{i}\right)=\lambda \mathcal{S}\left(\sum_{i} p_{i} \mathcal{Q}_{i}\right) \\
& =\lambda \mathcal{S}(\mathcal{Q})=\lambda^{\prime} \mathcal{S}\left(\mathcal{Q}^{\prime}\right)=\lambda^{\prime} \mathcal{S}\left(\sum_{j} p_{j}^{\prime} \mathcal{Q}_{j}^{\prime}\right) \\
& =\lambda^{\prime} \sum_{j} p_{j}^{\prime} \mathcal{S}\left(\mathcal{Q}_{j}^{\prime}\right)=\sum_{j} \lambda_{j}^{\prime} \mathcal{S}\left(\mathcal{Q}_{j}^{\prime}\right) .
\end{aligned}
$$

Hence, the map $\mathcal{S}$ can be uniquely extended to a map $\mathcal{S}_{\text {lin }}$ transforming the cone

$$
O p_{+}\left(\mathcal{H}_{\text {in }}, \mathcal{H}_{\text {out }}\right):=\left\{\lambda \mathcal{Q} \mid \mathcal{Q} \in \operatorname{Op}\left(\mathcal{H}_{\text {in }}, \mathcal{H}_{\text {out }}\right), \lambda \geqslant 0\right\}
$$

into the cone

$$
\mathrm{Op}_{+}\left(\mathcal{K}_{\text {in }}, \mathcal{K}_{\text {out }}\right):=\left\{\lambda \mathcal{Q} \mid \mathcal{Q} \in \operatorname{Op}\left(\mathcal{K}_{\text {in }}, \mathcal{K}_{\text {out }}\right), \lambda \geqslant 0\right\} .
$$

and the map $\mathcal{S}_{\text {lin }}$ is linear on conic combinations.

To conclude, we invoke the fact that every map from a cone $\mathrm{C}$ to a cone $\mathrm{C}^{\prime}$ that is linear on conic combinations can be uniquely extended to a linear map from the linear span of $C$ to the linear span of $C^{\prime}$ [91]. Since the linear span of the cone of quantum operations $\mathrm{Op}_{+}\left(\mathcal{H}_{\text {in }}, \mathcal{H}_{\text {out }}\right)$ is the space of all maps $\operatorname{Map}\left(\mathcal{H}_{\text {in }}, \mathcal{H}_{\text {out }}\right)$, this concludes the proof.

Since the linear extension is unique, in the following we will drop the subscript from $\mathcal{S}_{\text {lin }}$, and simply write $\mathcal{S}$.

\section{b. Choi representation of linear maps and supermaps}

The other key ingredient in the proof of Theorem 3 is the Choi representation of linear maps [80], widely used in quantum information theory [92-95] and quantum foundations $[59,96]$. The Choi representation of a map $\mathcal{M}: L\left(\mathcal{H}_{\text {in }}\right) \rightarrow$ $L\left(\mathcal{H}_{\text {out }}\right)$ is the linear operator $\operatorname{Choi}(\mathcal{M}) \in L\left(\mathcal{H}_{\text {out }} \otimes \mathcal{H}_{\text {in }}\right)$ defined by

$$
\operatorname{Choi}(\mathcal{M}):=\sum_{i, j} \mathcal{M}(|i\rangle\langle j|) \otimes|i\rangle\langle j|
$$


where $\{|i\rangle\}$ is a fixed (but otherwise arbitrary) basis for the input Hilbert space $\mathcal{H}_{\text {in }}$.

The Choi representation is related to the Jamiołkowski representation [97]

$$
\operatorname{Jam}(\mathcal{M}):=\sum_{i, j} \mathcal{M}(|i\rangle\langle j|) \otimes|j\rangle\langle i|,
$$

the difference between the two representations being a partial transpose operation on the input Hilbert space.

For a quantum operation $\mathcal{Q}$, the Choi operator is proportional to an unnormalized state, namely $\operatorname{Choi}(\mathcal{Q}) / d_{\text {in }}$. The relation between the set of quantum maps and the set of bipartite states was investigated in Refs. [94,95], and in Ref. [59], which extended the correspondence to a class of physical theories including quantum theory as a special case.

The Choi representation offers a convenient way to represent supermaps. In the Choi representation, a supermap $\mathcal{S}$ : $\operatorname{Map}\left(\mathcal{H}_{\text {in }}, \mathcal{H}_{\text {out }}\right) \rightarrow \operatorname{Map}\left(\mathcal{K}_{\text {in }}, \mathcal{K}_{\text {out }}\right)$ is associated to a linear $\operatorname{map} \widehat{\mathcal{S}}: L\left(\mathcal{H}_{\text {out }} \otimes \mathcal{H}_{\text {in }}\right) \rightarrow L\left(\mathcal{K}_{\text {out }} \otimes \mathcal{K}_{\text {in }}\right)$ uniquely defined by

$$
\widehat{\mathcal{S}}(\operatorname{Choi}(\mathcal{M})):=\operatorname{Choi}(\mathcal{S}(\mathcal{M})) \quad \forall \mathcal{M} \in \operatorname{Map}(\mathcal{H}) .
$$

\section{c. Derivation of the theorem}

The first step in the proof of Theorem 3 is to show that the map $\widehat{\mathcal{S}}$ is a symmetry of quantum states:

Lemma 7. For every symmetry of quantum operations $\mathcal{S}: \operatorname{Op}\left(\mathcal{H}_{\text {in }}, \mathcal{H}_{\text {out }}\right) \rightarrow \operatorname{Op}\left(\mathcal{K}_{\text {in }}, \mathcal{K}_{\text {out }}\right)$, the associated map $\widehat{\mathcal{S}}:$ $\operatorname{St}\left(\mathcal{H}_{\text {out }} \otimes \mathcal{H}_{\text {in }}\right) \rightarrow \operatorname{St}\left(\mathcal{K}_{\text {out }} \otimes \mathcal{K}_{\text {in }}\right)$ is a state space symmetry.

In order not to break the flow of the argument, we postpone the proof of Lemma 7 to Appendix E.

An immediate consequence of Lemma 7 is that the total dimension of the spaces $\mathcal{H}_{\text {out }} \otimes \mathcal{H}_{\text {in }}$ and $\mathcal{K}_{\text {out }} \otimes \mathcal{K}_{\text {in }}$ must be the same, namely,

$$
d_{\mathcal{H}_{\text {in }}} d_{\mathcal{H}_{\text {out }}}=d_{\mathcal{K}_{\text {in }}} d_{\mathcal{K}_{\text {out }}} .
$$

The second step in the proof of Theorem 3 is to break down the operation space symmetry $\mathcal{S}$ into two state space symmetries. This result is accomplished through the following lemma.

Lemma 8. For every quantum operation symmetry $\mathcal{S}$ : $\operatorname{Map}\left(\mathcal{H}_{\text {in }}, \mathcal{H}_{\text {out }}\right) \rightarrow \operatorname{Map}\left(\mathcal{K}_{\text {in }}, \mathcal{K}_{\text {out }}\right)$, there exists a state space symmetry $\mathcal{J}: L\left(\mathcal{H}_{\text {in }}\right) \rightarrow L\left(\mathcal{K}_{\text {in }}\right)$ such that

$$
\widehat{\mathcal{S}}\left(I_{\mathcal{H}_{\text {out }}} \otimes \rho\right)=I_{\mathcal{K}_{\text {out }}} \otimes \mathcal{J}(\rho) \quad \forall \rho \in \operatorname{St}\left(\mathcal{H}_{\text {in }}\right) .
$$

If $\widehat{\mathcal{S}}$ is unitary, then $\mathcal{J}$ is unitary. If $\widehat{\mathcal{S}}$ is antiunitary, then $\mathcal{J}$ is antiunitary.

The proof of Lemma 8 can be found in Appendix F. A consequence of Lemma 8 is that the input spaces $\mathcal{H}_{\text {in }}$ and $\mathcal{K}_{\text {in }}$ have the same dimension. Combining this fact with Eq. (D10), we obtain that also the output spaces $\mathcal{H}_{\text {out }}$ and $\mathcal{K}_{\text {out }}$ must have the sam dimension. In summary,

$$
\begin{aligned}
& d_{\mathcal{H}_{\text {in }}}=d_{\mathcal{K}_{\text {in }}}:=d_{\text {in }} \\
& d_{\mathcal{H}_{\text {out }}}=d_{\mathcal{K}_{\text {out }}}:=d_{\text {out }} .
\end{aligned}
$$

The Wigner theorem for quantum operations is then obtained by combining lemmas 7 and 8 .

Proof of Theorem 3. Let $\mathcal{J}: \operatorname{St}\left(\mathcal{H}_{\text {in }}\right) \rightarrow \operatorname{St}\left(\mathcal{K}_{\text {in }}\right)$ be the state space symmetry defined in Lemma 8 . The goal of the proof is to show that $\widehat{\mathcal{S}}$ can be broken down into the product of two state space symmetries.

Consider first the case where both $\widehat{\mathcal{S}}$ and $\mathcal{J}$ are unitary transformations. Then, write the maximally mixed state $I_{\mathcal{H}_{\text {out }}} / d_{\text {out }}$ as the marginal of the maximally entangled state $\left|\phi_{+}\right\rangle=\sum_{i}|i\rangle \otimes|i\rangle / \sqrt{d_{\text {out }}} \in \mathcal{H}_{R} \otimes \mathcal{H}_{\text {out }}$, where $\mathcal{H}_{R}$ is an auxiliary Hilbert space, isomorphic to $\mathcal{H}_{\text {out }}$. Using the notation $P_{\psi}:=|\psi\rangle\langle\psi|$ for a generic vector $\psi$, we can rewrite Eq. (D11) as

$$
\left(\operatorname{Tr}_{R} \otimes \widehat{\mathcal{S}}\right)\left(P_{\phi_{+}} \otimes \rho\right)=\left(\operatorname{Tr}_{R} \otimes \mathcal{I}_{\mathcal{K}_{\text {out }}} \otimes \mathcal{J}\right)\left(P_{\phi_{+}} \otimes \rho\right) .
$$

Since Eq. (D11) holds for every density matrix $\rho$, it holds in particular for every pure state. Hence, we have

$$
\left(\operatorname{Tr}_{R} \otimes \widehat{\mathcal{S}}\right)\left(P_{\phi_{+}} \otimes P_{\psi}\right)=\left(\operatorname{Tr}_{R} \otimes \mathcal{I}_{\mathcal{K}_{\text {out }}} \otimes \mathcal{J}\right)\left(P_{\phi_{+}} \otimes P_{\psi}\right),
$$

for every unit vector $\psi \in \mathcal{H}_{\text {in }}$.

Since $\widehat{\mathcal{S}}$ and $\mathcal{J}$ are unitary transformations, the two operators on the two sides of the equality are rank-one projectors, representing pure states. Equation (D14) shows that these two pure states have the same marginal states once the auxiliary system is traced out. In other words, they are two purifications of the same mixed state. Hence, the two pure states must be equivalent up to a unitary transformation on the auxiliary system. Explicitly, one must have

$$
\left(\mathcal{I}_{R} \otimes \widehat{\mathcal{S}}\right)\left(P_{\phi_{+}} \otimes P_{\psi}\right)=\left(\mathcal{W}_{\psi} \otimes \mathcal{I}_{\mathcal{K}_{\text {out }}} \otimes \mathcal{J}\right)\left(P_{\phi_{+}} \otimes P_{\psi}\right),
$$

for some unitary transformation $\mathcal{W}_{\psi}: L\left(\mathcal{H}_{R}\right) \rightarrow L\left(\mathcal{H}_{R}\right)$, possibly depending on $\psi$.

Now, we use the relation $(X \otimes I)\left|\phi_{+}\right\rangle=\left(I \otimes X^{T}\right)\left|\phi_{+}\right\rangle$, valid for every operator $X \in L\left(\mathcal{H}_{R}\right) \simeq L\left(\mathcal{H}_{\text {out }}\right)$. Using this relation, Eq. (D15) can be rewritten as

$$
\left(\mathcal{I}_{R} \otimes \widehat{\mathcal{S}}\right)\left(P_{\phi_{+}} \otimes P_{\psi}\right)=\left(\mathcal{I}_{R} \otimes \mathcal{W}_{\psi}^{T} \otimes \mathcal{J}\right)\left(P_{\phi_{+}} \otimes P_{\psi}\right)
$$

where $\mathcal{W}_{\psi}^{T}$ is the transpose of $\mathcal{W}_{\psi}$.

From the above equation we obtain

$$
\widehat{\mathcal{S}}\left(P_{\varphi} \otimes P_{\psi}\right)=\mathcal{W}_{\psi}^{T}\left(P_{\varphi}\right) \otimes \mathcal{J}\left(P_{\psi}\right)
$$

for every pair of unit vectors $\varphi$ and $\psi$.

Since the transformations on both sides of the equality are unitary, it is easy to show that the transformation $\mathcal{W}_{\psi}$ must be independent of $\psi$ (see Appendix $\mathrm{G}$ for an explicit proof). Hence, Eq. (D17) becomes $\widehat{\mathcal{S}}\left(P_{\varphi} \otimes P_{\psi}\right)=\mathcal{W}^{T}\left(P_{\varphi}\right) \otimes \mathcal{J}\left(P_{\psi}\right)$, $\forall \varphi, \forall \psi$ or equivalently, $\widehat{\mathcal{S}}=\mathcal{W}^{T} \otimes \mathcal{J}$.

Translating back from the Choi representation, we then obtain that the map $\mathcal{S}$ has the form $\mathcal{S}(\mathcal{Q})=\mathcal{W}^{T} \circ \mathcal{Q} \circ \mathcal{J}^{T}$. Defining $\mathcal{S}_{1}:=\mathcal{J}^{T}$ and $\mathcal{S}_{2}:=\mathcal{W}^{T}$ we then obtain the desired result.

To conclude the proof, consider the case where the state space symmetries $\widehat{\mathcal{S}}$ and $\mathcal{J}$ are both antiunitary. In this case, we can rewrite them as $\widehat{\mathcal{S}}=\widehat{\mathcal{S}}^{\prime} \circ\left(\mathcal{T}_{\mathcal{H}_{\text {out }}} \otimes \mathcal{T}_{\mathcal{H}_{\text {in }}}\right)$ and $\mathcal{J}=$ $\mathcal{J}^{\prime} \circ \mathcal{T}_{\mathcal{H}_{\text {in }}}$, where $\widehat{\mathcal{S}}^{\prime}$ and $\mathcal{J}^{\prime}$ are unitary transformations, and $\mathcal{T}_{\mathcal{H}_{\text {in }}}\left(\mathcal{T}_{\mathcal{H}_{\text {out }}}\right)$ is the transpose map on $\mathcal{H}_{\text {in }}\left(\mathcal{H}_{\text {out }}\right)$. Inserting the expression of $\widehat{\mathcal{S}}$ and $\mathcal{J}$ into Eq. (D11), we obtain that Eq. (D17) holds also for $\widehat{\mathcal{S}}^{\prime}$ and $\mathcal{J}^{\prime}$.

Hence, the same derivation used in the unitary case holds for the maps $\widehat{\mathcal{S}}^{\prime}$ and $\mathcal{J}^{\prime}$, and we obtain $\widehat{\mathcal{S}}^{\prime}=\mathcal{W}^{\prime T} \otimes$ $\mathcal{J}^{\prime}$, for a suitable unitary transformation $\mathcal{W}^{\prime}$. Getting back 
to the map $\widehat{\mathcal{S}}$, we obtain $\widehat{\mathcal{S}}=\widehat{\mathcal{S}}^{\prime} \circ\left(\mathcal{T}_{\mathcal{H}_{\text {out }}} \otimes \mathcal{T}_{\mathcal{H}_{\text {in }}}\right)=\left(\mathcal{W}^{\prime T} \circ\right.$ $\left.\mathcal{T}_{\mathcal{H}_{\text {out }}}\right) \otimes\left(\mathcal{J}^{\prime} \circ \mathcal{T}_{\mathcal{H}_{\text {in }}}\right)=\mathcal{S}_{2} \otimes \mathcal{J}$, where $\mathcal{S}_{2}:=\mathcal{W}^{\prime T} \circ \mathcal{T}_{\mathcal{H}_{\text {out }}}$ is an antiunitary symmetry.

Translating back from the Choi representation, we then obtain that the map $\mathcal{S}$ has the form $\mathcal{S}(\mathcal{Q})=\mathcal{S}_{2} \circ \mathcal{Q} \circ \mathcal{S}_{1}$, where $\mathcal{S}_{1}:=\mathcal{J}^{T}$ is an antiunitary transformation.

\section{APPENDIX E: PROOF OF LEMMA 7}

The proof uses the notion of channel-preserving homomorphism, that is, a supermap that maps quantum channels into quantum channels [8-11].

Definition 5. A supermap $\quad \mathcal{S}: \operatorname{Op}\left(\mathcal{H}_{\text {in }}, \mathcal{H}_{\text {out }}\right) \rightarrow$ $\operatorname{Op}\left(\mathcal{K}_{\text {in }}, \mathcal{K}_{\text {out }}\right)$ is a homomorphism of quantum operations if it preserves convex combinations and the null operation. An homomorphism $\mathcal{S}$ is channel-preserving if, for every channel $\mathcal{C} \in \operatorname{Chan}\left(\mathcal{H}_{\text {in }}, \mathcal{H}_{\text {out }}\right)$, one has $\mathcal{S}(\mathcal{C}) \in \operatorname{Chan}\left(\mathcal{K}_{\text {in }}, \mathcal{K}_{\text {out }}\right)$.

We now show that every channel-preserving homomorphism induces a homomorphism of quantum states.

Lemma 9. Let $\mathcal{S}: \operatorname{Op}\left(\mathcal{H}_{\text {in }}, \mathcal{H}_{\text {out }}\right) \rightarrow \operatorname{Op}\left(\mathcal{K}_{\text {in }}, \mathcal{K}_{\text {out }}\right)$ be a channel-preserving homomorphism, let $\widehat{\mathcal{S}}: L\left(\mathcal{H}_{\text {out }} \otimes \mathcal{H}_{\text {in }}\right) \rightarrow$ $L\left(\mathcal{K}_{\text {out }} \otimes \mathcal{K}_{\text {in }}\right)$ be the associated map in the Choi representation. Then, there exists a state space homomorphism $\mathcal{A}$ : $\operatorname{St}\left(\mathcal{K}_{\text {in }}\right) \rightarrow \operatorname{St}\left(\mathcal{H}_{\text {in }}\right)$ such that

$$
\widehat{\mathcal{S}}^{\dagger}\left(I_{\mathcal{K}_{\text {out }}} \otimes \rho\right)=I_{\mathcal{H}_{\text {in }}} \otimes \mathcal{A}(\rho) \quad \forall \rho \in \operatorname{St}\left(\mathcal{K}_{\text {in }}\right),
$$

where $\widehat{\mathcal{S}}^{\dagger}$ is the adjoint of the map $\widehat{\mathcal{S}}$. If $\mathcal{S}$ is a symmetry of quantum operations, then $\mathcal{A}$ is a symmetry of quantum states.

Proof. Let us define the set ChoiChan $\left(\mathcal{H}_{\text {in }}, \mathcal{H}_{\text {out }}\right)$ to be the set of Choi operators of quantum channels in $\operatorname{Chan}\left(\mathcal{H}_{\text {in }}, \mathcal{H}_{\text {out }}\right)$. For a non-negative operator $A \in$ $L\left(\mathcal{H}_{\text {out }} \otimes \mathcal{H}_{\text {in }}\right)$, the relation $\langle A, C\rangle=1$ holds for every $C \in$ ChoiChan $\left(\mathcal{H}_{\text {in }}, \mathcal{H}_{\text {out }}\right)$ if and only if $A$ has the form $A=$ $I_{\text {out }} \otimes \rho$ for some density matrix $\rho \in \operatorname{St}\left(\mathcal{H}_{\text {in }}\right)$ [98]. Now, for every density matrix $\rho \in \operatorname{St}\left(\mathcal{K}_{\text {in }}\right)$, the operator $\widehat{\mathcal{S}}^{\dagger}\left(I_{\mathcal{K}_{\text {out }}} \otimes \rho\right)$ is non-negative and satisfies

$$
\begin{aligned}
\left\langle\widehat{S}^{\dagger}\left(I_{\mathcal{K}_{\text {out }}} \otimes \rho\right), C\right\rangle & =\left\langle I_{\mathcal{H}_{\text {out }}} \otimes \rho, \widehat{S}(C)\right\rangle \\
& =1,
\end{aligned}
$$

the last equality following from the fact that $\widehat{\mathcal{S}}(C)$ is in ChoiChan $\left(\mathcal{K}_{\text {in }}, \mathcal{K}_{\text {out }}\right)$, because $\mathcal{S}$ is channel-preserving. Hence, $\widehat{\mathcal{S}}^{\dagger}\left(I_{\mathcal{K}_{\text {out }}} \otimes \rho\right)$ must be of the form $I_{\mathcal{H}_{\text {out }}} \otimes \rho^{\prime}$ for some density matrix $\rho^{\prime} \in \operatorname{St}\left(\mathcal{H}_{\text {in }}\right)$. Explicitly, the density matrix $\rho^{\prime}$ can be computed as

$$
\begin{aligned}
\rho^{\prime} & :=\frac{\operatorname{Tr}_{1}\left[\widehat{\mathcal{S}}^{\dagger}\left(I_{\mathcal{K}_{\text {out }}} \otimes \rho\right)\right]}{d} \\
& =: \mathcal{A}(\rho),
\end{aligned}
$$

where $\operatorname{Tr}_{1}$ denotes the partial trace over the first Hilbert space. The map $\mathcal{A}$ transforms density matrices into density matrices, and it preserves convex combinations. Therefore it is a state space homomorphism.

Now, suppose that $\mathcal{S}$ is a symmetry of quantum operations. Let $\widehat{\mathcal{T}}:=\mathcal{\mathcal { S }}^{-1}$ be the inverse of $\widehat{\mathcal{S}}$, and let $\mathcal{B}: \operatorname{St}\left(\mathcal{H}_{\text {in }}\right) \rightarrow$ $\operatorname{St}\left(\mathcal{K}_{\text {in }}\right)$ be the state space homomorphism associated to $\widehat{\mathcal{T}}$ as in Lemma 9. Hence, for every density matrix $\rho \in$ $\operatorname{St}\left(\mathcal{K}_{\text {in }}\right)$, one has $I_{\mathcal{K}_{\text {out }}} \otimes \rho=\left(\widehat{\mathcal{T}}^{\dagger} \circ \widehat{\mathcal{S}}^{\dagger}\right)\left(I_{\mathcal{K}_{\text {out }}} \otimes \rho\right)=I_{\mathcal{K}_{\text {out }}} \otimes$
$(\mathcal{B} \circ \mathcal{A})(\rho)$. Hence, $\mathcal{B} \circ \mathcal{A}=\mathcal{I}_{\mathcal{K}_{\text {in }}}$. Similarly, one can prove that $\mathcal{A} \circ \mathcal{B}=\mathcal{I}_{\mathcal{H}_{\text {in }}}$. Hence, $\mathcal{A}$ is a state space symmetry.

Equipped with the above result, we are ready to prove Lemma 7.

Proof of Lemma 7. We need to show that the map $\widehat{\mathcal{S}}$ is a state space symmetry whenever $\mathcal{S}$ is a symmetry of quantum operations. We first show that $\widehat{\mathcal{S}}^{\dagger}$ is a unital map, namely $\widehat{\mathcal{S}}^{\dagger}\left(I_{\mathcal{K}_{\text {out }}} \otimes I_{\mathcal{K}_{\text {in }}}\right)=I_{\mathcal{H}_{\text {out }}} \otimes I_{\mathcal{H}_{\text {in }}}$. By Lemma 9 , we have $\widehat{\mathcal{S}}^{\dagger}\left(I_{\mathcal{K}_{\text {out }}} \otimes I_{\mathcal{K}_{\text {in }}}\right)=I_{\mathcal{H}_{\text {out }}} \otimes \mathcal{A}\left(I_{\mathcal{K}_{\text {in }}}\right)$, where $\mathcal{A}$ is a state space symmetry. By Wigner's theorem, every state space symmetry is either unitary or antiunitary, and therefore it is unital. Hence, $\mathcal{A}\left(I_{\mathcal{K}_{\text {in }}}\right)=I_{\mathcal{H}_{\text {in }}}$, and $\widehat{\mathcal{S}}^{\dagger}\left(I_{\mathcal{K}_{\text {out }}} \otimes I_{\mathcal{K}_{\text {in }}}\right)=I_{\mathcal{H}_{\text {out }}} \otimes$ $I_{\mathcal{H}_{\text {in }}}$. This proves that $\widehat{\mathcal{S}}^{\dagger}$ is unital.

Moreover, the map $\widehat{\mathcal{S}}^{\dagger}$ is positive: for every positive operator $P$ and for every unit vector $\Psi \in \mathcal{H}_{\text {out }} \otimes$ $\mathcal{H}_{\text {in }}$ one has $\left\langle\Psi\left|\widehat{\mathcal{S}}^{\dagger}(P)\right| \Psi\right\rangle=\left\langle\widehat{\mathcal{S}}^{\dagger}(P), P_{\Psi}\right\rangle=\left\langle P, \widehat{\mathcal{S}}\left(P_{\Psi}\right)=\right.$ $\operatorname{Tr}\left[P \widehat{\mathcal{S}}\left(P_{\Psi}\right)\right] \geqslant 0$, because $\widehat{\mathcal{S}}\left(P_{\psi}\right)$ is a positive operator. This proves that $\widehat{\mathcal{S}}^{\dagger}$ is positive.

Since $\widehat{\mathcal{S}}^{\dagger}$ is positive and unital, Lemma 4 implies that $\widehat{\mathcal{S}}$ is a state space homomorphism. Applying the same argument to the inverse map $\widehat{\mathcal{S}}^{-1}$ we obtain that $\widehat{\mathcal{S}}$ is a state space symmetry.

\section{APPENDIX F: PROOF OF LEMMA 8}

We need to prove that for every operation space symmetry $\mathcal{S}$ there exists a state space symmetry $\mathcal{J}$ such that the condition

$$
\widehat{\mathcal{S}}\left(I_{\mathcal{H}_{\text {out }}} \otimes \rho\right)=I_{\mathcal{K}_{\text {out }}} \otimes \mathcal{J}(\rho) \quad \forall \rho \in \operatorname{St}\left(\mathcal{H}_{\text {in }}\right),
$$

and the symmetry $\mathcal{J}$ is unitary (antiunitary) whenever the symmetry $\widehat{\mathcal{S}}$ is unitary (antiunitary).

Note that (1) the map $\widehat{\mathcal{S}}$ is a state space symmetry (Lemma 7), and (2) Lemma 9 already guarantees the existence of a state space symmetry $\mathcal{A}$ such that

$$
\widehat{\mathcal{S}}^{\dagger}\left(I_{\mathcal{K}_{\text {out }}} \otimes \rho\right)=I_{\mathcal{H}_{\text {in }}} \otimes \mathcal{A}(\rho) \quad \forall \rho \in \operatorname{St}\left(\mathcal{K}_{\text {in }}\right) .
$$

Since $\widehat{\mathcal{S}}$ and $\mathcal{A}$ are state space symmetries, Wigner's theorem implies that they are either unitary or antiunitary transformations. In either case, one has $\widehat{\mathcal{S}}^{\dagger} \widehat{\mathcal{S}}=\mathcal{I}_{\mathcal{H}_{\text {out }}} \otimes \mathcal{I}_{\mathcal{H}_{\text {in }}}$ and $\mathcal{A}^{\dagger} \mathcal{A}=\mathcal{I}_{\mathcal{K}_{\text {in }}}$. Hence, Eq. (F2) implies Eq. (F1) with $\mathcal{J}=\mathcal{A}^{\dagger}$.

It only remains to show that $\mathcal{J}$ is unitary (antiunitary) whenever $\widehat{\mathcal{S}}$ is unitary (antiunitary). To this purpose, note that Eq. (F1) implies

$$
\mathcal{J}(\rho)=\frac{\operatorname{Tr}_{\mathcal{K}_{\text {out }}}\left[\widehat{\mathcal{S}}\left(I_{\mathcal{H}_{\text {out }}} \otimes \rho\right)\right]}{d_{\text {out }}} \quad \forall \rho \in \operatorname{St}\left(\mathcal{H}_{\text {in }}\right) .
$$

If $\widehat{\mathcal{S}}$ is unitary, then the map acting on $\rho$ on the right-hand side is completely positive. Hence, the map $\mathcal{J}$ is completely positive, which is possible only if $\mathcal{J}$ is unitary.

Similarly, if $\widehat{\mathcal{S}}$ is antiunitary, the composition of the map on the right-hand side with the transpose map $\mathcal{T}_{\mathcal{H}_{\text {in }}}$ is completely 
positive. Hence, the map $\mathcal{J} \circ \mathcal{T}_{\mathcal{H}_{\text {in }}}$ is completely positive, which is possible only if $\mathcal{J}$ is antiunitary.

\section{APPENDIX G: PROOF THAT THE UNITARY TRANSFORMATION $\mathcal{W}_{\psi}$ IN EQ. (D17) IS INDEPENDENT OF $\psi$}

We provide two alternative proofs, the first based on a quantum information-theoretic argument, and the second on explicit matrix calculations.

Quantum information-theoretic proof. Equation (D17) states that the unitary transformations $\widehat{\mathcal{S}}, \mathcal{W}_{\psi}$, and $\mathcal{J}$ satisfy the condition

$$
\widehat{\mathcal{S}}\left(P_{\varphi} \otimes P_{\psi}\right)=\mathcal{W}_{\psi}^{T}\left(P_{\varphi}\right) \otimes \mathcal{J}\left(P_{\psi}\right)
$$

for every unit vector $\varphi$. A physical way to read the equation is to imagine that the reversible process $\widehat{\mathcal{S}}$ is applied to a system, initially in the state $P_{\psi}$, coupled to an ancilla in the state $P_{\varphi}$. The result of the process is to implement a fixed unitary transformation $\mathcal{J}$ on the system, and another unitary transformation $\mathcal{W}_{\psi}$ on the ancilla. Since the transformation $\mathcal{J}$ is unitary, it can be reversed by applying the inverse process $\mathcal{J}^{-1}$. If this is done, the system is brought back to the state $P_{\psi}$, while the ancilla is in a new state $\mathcal{W}_{\psi}\left(P_{\varphi}\right)$, possibly depending on $\psi$. However, the so-called "No-informationwithout-disturbance" theorem implies that the state of the ancilla should be independent of $\psi$, for otherwise one could extract information about $\psi$ by measuring the ancilla, without affecting the state of the system (see Ref. [99] for a general proof of the no-information-without-disturbance theorem, which does not require the details of the quantum framework). Hence, we conclude that, for every state $P_{\varphi}$, the state $\mathcal{W}_{\psi}^{T}(\varphi)$ should be independent of $\psi$. In other words, the transformation $\mathcal{W}_{\psi}^{T}$ should be independent of $\psi$.

Matrix algebra proof. Let $\psi$ and $\psi^{\prime}$ be two different unit vectors. Since the transformation $\widehat{\mathcal{S}}$ is unitary, we have

$$
\begin{aligned}
\left\langle\widehat{\mathcal{S}}\left(P_{\varphi} \otimes P_{\psi}\right), \widehat{\mathcal{S}}\left(P_{\varphi} \otimes P_{\psi^{\prime}}\right)\right\rangle & =\left\langle P_{\varphi} \otimes P_{\psi}, P_{\varphi} \otimes P_{\psi^{\prime}}\right\rangle \\
& =\left\langle P_{\psi}, P_{\psi^{\prime}}\right\rangle .
\end{aligned}
$$

Using Eq. (D17) for $\psi$ and $\psi^{\prime}$, we obtain

$$
\begin{aligned}
\left\langle P_{\psi}, P_{\psi}^{\prime}\right\rangle & =\left\langle\widehat{\mathcal{S}}\left(P_{\varphi} \otimes P_{\psi}\right), \widehat{\mathcal{S}}\left(P_{\varphi} \otimes P_{\psi^{\prime}}\right)\right\rangle \\
& =\left\langle\mathcal{W}_{\psi}^{T}\left(P_{\varphi}\right) \otimes \mathcal{J}\left(P_{\psi}\right), \mathcal{W}_{\psi^{\prime}}^{T}\left(P_{\varphi}\right) \otimes \mathcal{J}\left(P_{\psi^{\prime}}\right)\right\rangle \\
& =\left\langle\mathcal{W}_{\psi}^{T}(\varphi), \mathcal{W}_{\psi^{\prime}}^{T}\left(P_{\varphi}\right)\right\rangle\left\langle\mathcal{J}\left(P_{\psi}\right), \mathcal{J}\left(P_{\psi^{\prime}}\right)\right\rangle \\
& =\left\langle P_{\varphi}, \overline{\mathcal{W}}_{\psi} \mathcal{W}_{\psi^{\prime}}^{T}\left(P_{\varphi}\right)\right\rangle\left\langle P_{\psi}, P_{\psi^{\prime}}\right\rangle,
\end{aligned}
$$

the last equation following from the fact that $\mathcal{J}$ is a state space symmetry, and therefore preserves the ray product.

Equation (G3) must hold for every choice of unit vectors $\varphi, \psi$, and $\psi^{\prime}$. Choosing $\psi$ and $\psi^{\prime}$ to be nonorthogonal, we have $\left\langle P_{\psi}, P_{\psi^{\prime}}\right\rangle \neq 0$, and Eq. (G3) becomes

$$
\left\langle P_{\varphi}, \overline{\mathcal{W}}_{\psi} \mathcal{W}_{\psi^{\prime}}^{T}\left(P_{\varphi}\right)\right\rangle=1 \quad \forall|\varphi\rangle \in \mathcal{H}_{\text {out }},
$$

which is equivalent to $\overline{\mathcal{W}}_{\psi} \mathcal{W}_{\psi^{\prime}}^{T}=\mathcal{I}_{\mathcal{H}_{\text {out }}}$. Hence, $\mathcal{W}_{\psi}=\mathcal{W}_{\psi^{\prime}}$ whenever $\psi$ and $\psi^{\prime}$ are nonorthogonal.

Now, for every two unit vectors $\psi_{1}$ and $\psi_{2}$ there is a third unit vector $\psi$ that is neither orthogonal to $\psi_{1}$ nor to $\psi_{2}$. Hence, one must have $\mathcal{W}_{\psi_{1}}=\mathcal{W}_{\psi}$ and $\mathcal{W}_{\psi_{2}}=\mathcal{W}_{\psi}$, which implies $\mathcal{W}_{\psi_{1}}=\mathcal{W}_{\psi_{1}}$. In summary, the unitary $\mathcal{W}_{\psi}$ is independent of $\psi$.

\section{APPENDIX H: DOUBLE ANTIUNITARY TRANSFORMATIONS ARE NOT COMPLETELY POSITIVE}

An active interpretation of double antiunitary operations is incompatible with a general notion of physical transformation, captured by the framework of quantum supermaps [8-11,18,19,26,33-35], also called quantum superchannels in later works [20-22,24,27,37,100,101] (see also Ref. [13] for a related notion of supermap transforming a certain set of completely positive maps into itself).

Quantum supermaps represent the most general transformations that can in principle be applied locally to the reduced dynamics of subsystems. Under such requirement, Ref. [8] showed that the most general quantum supermap on the set of quantum oeprations consists in the insertion of the input quantum operation $\mathcal{Q}$ between two quantum channels, $\mathcal{A}$ and $\mathcal{B}$, thus obtaining the new quantum operation $\mathcal{Q}^{\prime}=\mathcal{B} \circ(\mathcal{Q} \otimes$ $\mathcal{I}_{A}$ ) $\circ \mathcal{A}$, where $\mathcal{I}_{A}$ denotes the identity channel on an auxiliary quantum system, output by channel $\mathcal{A}$, input into channel $\mathcal{B}$, and unaffected by the quantum operation $\mathcal{Q}$. This result was later extended to infinite dimensions in Ref. [11].

Our Wigner theorem for quantum operations implies that the only reversible quantum supermaps are those for which the channels $\mathcal{A}$ and $\mathcal{B}$ are unitary, and the auxiliary system $A$ is trivial (i.e., one-dimensional).

The same conclusion was reached by Ref. [17] for quantum supermaps that are generated by a continuous-time evolution. Our Wigner theorem shows that the assumption of continuity is not necessary in the case of quantum supermaps on quantum operations: reversibility and the applicability to subsystems are already enough to single out the double unitary form for any transformation of quantum operations that can be implemented actively in standard quantum mechanics.

In contrast, double antiunitary symmetries cannot be applied locally to the evolution of subsystems. Mathematically, they are associated to linear maps that are not completely positive. In the following, we provide a proof of this fact.

First, note that every double antiunitary transformations is unitarily equivalent to the double transpose map $\tau_{\text {in/out }}$ : $\mathcal{Q} \mapsto \tau_{\text {out }} \circ \mathcal{Q} \circ \tau_{\text {in. }}$. Since unitary equivalence just amounts to a change of basis, in the following we will restrict our attention to the map $\tau_{\text {in/out }}$.

Under the action of the double transpose, a generic quantum operation $\mathcal{Q}$ with Kraus operators $\left\{\mathcal{Q}_{i}\right\}$ is transformed into a quantum operation $\mathcal{Q}^{\prime}$ with Kraus operators $\left\{\mathcal{Q}_{i}^{*}\right\}$, obtained by complex conjugation in a fixed basis.

Now, let us examine the transformation $\mathcal{Q} \mapsto \mathcal{Q}^{\prime}$ in the Choi representation [80]. The Choi operator of $\mathcal{Q}$ is

$$
\begin{aligned}
\operatorname{Choi}(\mathcal{Q}) & :=\sum_{m, n} \mathcal{Q}(|m\rangle\langle n|) \otimes|m\rangle\langle n| \\
& =\sum_{i}\left(Q_{i} \otimes I_{\mathcal{H}_{\text {in }}}\right)\left|I_{\mathcal{H}_{\text {in }}}\right\rangle\left\langle\left\langle\left\langle I_{\mathcal{H}_{\text {in }}}\right|\left(Q_{i}^{\dagger} \otimes I\right),\right.\right.
\end{aligned}
$$


where we used the notation $\left|I_{\mathcal{H}_{\text {in }}}\right\rangle:=\sum_{m}|m\rangle \otimes|m\rangle$. The Choi operator of $\mathcal{Q}^{\prime}$ is

$$
\begin{aligned}
\operatorname{Choi}\left(\mathcal{Q}^{\prime}\right) & \left.=\sum_{i}\left(Q_{i}^{*} \otimes I_{\mathcal{H}_{\text {in }}}\right)\left|I_{\mathcal{H}_{\text {in }}}\right\rangle\right\rangle\left\langle\left\langle I_{\mathcal{H}_{\text {in }}}\right|\left(Q_{i}^{T} \otimes I\right)\right. \\
& =[\operatorname{Choi}(\mathcal{Q})]^{*} \\
& =[\operatorname{Choi}(\mathcal{Q})]^{T},
\end{aligned}
$$

the last equality following from the fact that $\operatorname{Choi}(\mathcal{Q})$ is self-adjoint. Hence, at the level of Choi operators, the double transpose map $\tau_{\text {in/out }}$ is just the transpose. Since the transpose is known to be not completely positive, we conclude that the double transpose map is not a completely positive transformation. Physically, the failure of complete positivity implies that it is not possible to apply the map $\tau_{\text {in/out }}$ on a generic bipartite quantum operation [8].

\section{APPENDIX I: CHOI REPRESENTATION OF THE TRANSFORMATIONS $\Theta$ AND $\Theta^{\prime}$}

Here we consider the transformations $\Theta: \mathcal{Q} \rightarrow \mathcal{Q}^{\dagger}$ and $\Theta^{\prime}: \mathcal{Q} \rightarrow \mathcal{Q}^{T}$, which are the natural extensions of the timereversal transformations $U \mapsto U^{\dagger}$ and $U \mapsto U^{T}$, defined at the level of unitary dynamics.

Let us consider first the Choi representation of the transformation $\Theta^{\prime}: \mathcal{Q} \rightarrow \mathcal{Q}^{T}$.

For generic completely positive map $\mathcal{Q}: L\left(\mathcal{H}_{\text {in }}\right) \rightarrow$ $L\left(\mathcal{H}_{\text {out }}\right)$, written in the Kraus representation $\mathcal{Q}(\rho):=$ $\sum_{i} Q_{i} \rho Q_{i}^{\dagger}$, the Choi operator is given by

$$
\begin{aligned}
\operatorname{Choi}(\mathcal{Q}) & :=\sum_{m, n} \mathcal{Q}(|m\rangle\langle n|) \otimes|m\rangle\langle n| \\
& =\sum_{i}\left(Q_{i} \otimes I_{\mathcal{H}_{\text {in }}}\right)\left|I_{\mathcal{H}_{\text {in }}}\right\rangle\left\langle\left\langle I_{\mathcal{H}_{\text {in }}}\right|\left(Q_{i}^{\dagger} \otimes I\right),\right.
\end{aligned}
$$

where we used the notation $\left.\left|I_{\mathcal{H}_{\text {in }}}\right\rangle\right\rangle:=\sum_{m}|m\rangle \otimes|m\rangle$.

Using the condition

$$
\left(A \otimes I_{\mathcal{H}_{\text {in }}}\right)\left|I_{\mathcal{H}_{\text {in }}}\right\rangle=\left(I_{\mathcal{H}_{\text {out }}} \otimes A^{T}\right)\left|I_{\mathcal{H}_{\text {out }}}\right\rangle,
$$

valid for every operator $A: \mathcal{H}_{\text {in }} \rightarrow H_{\text {out }}$, we obtain the relation

$$
\begin{aligned}
\operatorname{Choi}(\mathcal{Q}) & =\sum_{i}\left(I_{\mathcal{H}_{\text {out }}} \otimes Q_{i}^{T}\right)\left|I_{\mathcal{H}_{\text {out }}}\right\rangle\left\langle\left( I_{\mathcal{H}_{\text {out }}} \mid\left(Q_{i}^{*} \otimes I_{\mathcal{H}_{\text {out }}}\right)\right.\right. \\
& =\operatorname{SWAP}_{\text {in/out }} \operatorname{Choi}\left(\mathcal{Q}^{T}\right) \operatorname{SWAP}_{\text {in } / \text { out }},
\end{aligned}
$$

where $\operatorname{SWAP}_{\text {in/out }}: \mathcal{H}_{\text {in }} \otimes \mathcal{H}_{\text {out }} \rightarrow \mathcal{H}_{\text {out }} \otimes \mathcal{H}_{\text {in }}$ is the swap operator, defined by the condition $\operatorname{SWAP}(\phi \otimes \psi)=\psi \otimes \phi$, $\forall \phi, \forall \psi$.

Hence, at the level of Choi operators, the map $\Theta^{\prime}: \mathcal{Q} \mapsto$ $\mathcal{Q}^{T}$ is represented by a unitary transformation. Explicitly, the transformation is

$$
\widehat{\Theta}^{\prime}: Q \mapsto \operatorname{SWAP}_{\text {out } / \text { in }} Q \operatorname{SWAP}_{\text {out } / \text { in }},
$$

with SWAP out $/$ in $_{\text {n }}:=\mathrm{SWAP}_{\text {in } / \text { out }}^{-1}$. This unitary transformation corresponds to the exchange between the input and output spaces of the Choi operator [81].
Let us now consider the Choi representation of the time reversal $\Theta: \mathcal{Q} \rightarrow \mathcal{Q}^{\dagger}$. By definition, we have

$$
\begin{aligned}
\operatorname{Choi}\left(\mathcal{Q}^{\dagger}\right) & =\sum_{i}\left(Q_{i}^{\dagger} \otimes I_{\mathcal{H}_{\text {in }}}\right)\left|I_{\mathcal{H}_{\text {in }}}\right\rangle\left\langle\left\langle I_{\mathcal{H}_{\text {in }}}\right|\left(Q_{i} \otimes I\right)\right. \\
& =\left[\sum_{i}\left(Q_{i}^{T} \otimes I_{\mathcal{H}_{\text {in }}}\right)\left|I_{\mathcal{H}_{\text {in }}}\right\rangle\left\langle\left\langle I_{\mathcal{H}_{\text {in }}}\right|\left(Q_{i}^{*} \otimes I\right)\right]^{*}\right. \\
& =\left[\operatorname{Choi}\left(\mathcal{Q}^{T}\right)\right]^{*} \\
& =\left[\operatorname{Choi}\left(\mathcal{Q}^{T}\right)\right]^{T} \\
& =\left[\operatorname{SWAP}_{\text {out } / \text { in }} Q \operatorname{SWAP}_{\text {out } / \text { in }}\right]^{T} .
\end{aligned}
$$

Hence, at the level of Choi representation, the map $\Theta^{\prime}: \mathcal{Q} \mapsto$ $\mathcal{Q}^{T}$ is represented by the map

$$
\widehat{\Theta}: Q \mapsto\left[\mathrm{SWAP}_{\text {out } / \text { in }} Q \mathrm{SWAP}_{\text {out } / \text { in }}\right]^{T},
$$

namely the antiunitary transformation resulting from the composition of the swap operation with the transpose.

\section{APPENDIX J: PROOF OF THEOREM 4}

The proof is by contradiction: we assume that the symmetry $\mathcal{S}$ exists, and show that its existence leads to a logical contradiction.

Let us start from the case of symmetries satisfying the condition $\mathcal{S}(\mathcal{U})=\mathcal{U}^{\dagger}$ for every unitary channel $\mathcal{U}$. Since $\mathcal{S}$ is symmetry of quantum operations, its action on a generic unitary channel $\mathcal{U}$ is either $\mathcal{S}(\mathcal{U})=\mathcal{W} \circ \mathcal{U} \circ \mathcal{V}$ or $\mathcal{S}(\mathcal{U})=$ $\mathcal{W} \circ \tau_{\text {in/out }}(\mathcal{U}) \circ \mathcal{V}$, where $\tau_{\text {in/out }}$ is the "double transpose".

Let us inspect these two cases separately. In the first case, the transformation $\mathcal{S}(\mathcal{U})=\mathcal{W} \circ \mathcal{U} \circ \mathcal{V}$ is a sequence of quantum channels. References [33,35] showed that the transformation $\mathcal{U} \mapsto \mathcal{U}^{\dagger}$ cannot be implemented by inserting the channel $\mathcal{U}$ in a sequence of quantum channels. For completeness, we provide an alternative proof here. The condition $\mathcal{W} \circ \mathcal{U} \circ \mathcal{V}=\mathcal{U}^{\dagger}$ implies $W U V=e^{i \gamma_{U}} U^{\dagger}$ for every unitary operator $U$. Choosing $U=I$ reveals that one should have $V=e^{i \gamma_{I}} W^{\dagger}$. Choosing $U=W$, one obtains $W e^{i \gamma_{I}}=e^{i \gamma_{W}} W^{\dagger}$. Hence, one must have $W^{\dagger} U e^{i \gamma_{W}} W^{\dagger}=e^{i \gamma_{U}} U^{\dagger}$, which implies that $W^{\dagger} U$ is proportional to $\left(W^{\dagger} U\right)^{\dagger}$. In other words, $W^{\dagger} U$ must be proportional to a Hermitian operator. But since $W^{\dagger} U$ is a generic unitary operator, this condition cannot be satisfied.

In the second case, the channel $\tau_{\text {in/out }}(\mathcal{U})$ has the form $\rho \mapsto U^{*} \rho U^{T}$. Hence, the condition $\mathcal{W} \circ \tau_{\text {in/out }}(\mathcal{U}) \circ \mathcal{V}=\mathcal{U}^{\dagger}$ implies $W \bar{U} V=e^{i \gamma_{U}} U^{\dagger}$ for every unitary operator $U$. Choosing $U=I$ reveals that one should have $V=e^{i \gamma_{l}} W^{\dagger}$. Choosing $U=\bar{W}$, one obtains $W e^{i \gamma_{I}}=e^{i \gamma_{\bar{W}}} W^{T}$. Hence, one must have $W^{T} \bar{U} e^{i \gamma_{W}} W^{\dagger}=e^{i \gamma_{U}} U^{\dagger}$, which implies that $W^{T} \bar{U}$ is proportional to $\left(W^{T} \bar{U}\right)^{T}$. In other words, $W^{T} \bar{U}$ must be proportional to a symmetric operator. But since $W^{T} \bar{U}$ is a generic unitary operator, this condition cannot be satisfied.

Summarising, no symmetry of the set of quantum operations can satisfy the condition $\mathcal{S}(\mathcal{U})=\mathcal{U}^{\dagger}$ for every unitary channel $\mathcal{U}$. Let us now consider symmetries satisfying the condition $\mathcal{S}(\mathcal{U})=\mathcal{U}^{T}$ for every unitary channel $\mathcal{U}$. Note that the relation $\mathcal{U}^{\dagger}=\tau_{\text {in/out }}\left(\mathcal{U}^{T}\right)$ holds for every unitary channel $\mathcal{U}$. If there existed a symmetry $\mathcal{S}$ such that $\mathcal{S}(\mathcal{U})=\mathcal{U}^{T}$ for every unitary channel, then one could combine the symmetry $\mathcal{S}$ with the double transpose, thus obtaining a symmetry $\mathcal{S}^{\prime}=$ $\tau_{\text {in/out }} \circ \mathcal{S}$ such that $\mathcal{S}^{\prime}(\mathcal{U})=\mathcal{U}^{\dagger}$ for every unitary channel, in contradiction with the previous part of the proof. 
[1] Hermann Weyl, The Theory of Groups and Quantum Mechanics (Dover, New York, 1950).

[2] Eugene Paul Wigner, Group Theory and its Application to the Quantum Mechanics of Atomic Spectra (Academic Press, New York, 1959).

[3] John F. Cornwell, Group Theory in Physics: An Introduction (Academic Press, New York, 1997).

[4] Hal Tasaki, Physics and Mathematics of Quantum Many-Body Systems (Springer International Publishing, 2020).

[5] Masahito Hayashi, A Group Theoretic Approach to Quantum Information (Springer International Publishing, 2017).

[6] Steven Weinberg, The Quantum Theory of Fields (Cambridge University Press, Cambridge, UK, 1995), Vol. 2.

[7] E. Wigner, Normal form of antiunitary operators, J. Math. Phys. 1, 409 (1960).

[8] G. Chiribella, G. M. D'Ariano, and P. Perinotti, Transforming quantum operations: quantum supermaps, Europhys. Lett. 83, 30004 (2008).

[9] G. Chiribella, G. M. D’Ariano, and P. Perinotti, Theoretical framework for quantum networks, Phys. Rev. A 80, 022339 (2009).

[10] G. Chiribella, G. M. D’Ariano, P. Perinotti, and B. Valiron, Quantum computations without definite causal structure, Phys. Rev. A 88, 022318 (2013).

[11] G. Chiribella, A. Toigo, and V. Umanità, Normal completely positive maps on the space of quantum operations, Open Systems Information Dynamics 20, 1350003 (2013).

[12] A. Bisio and P. Perinotti, Theoretical framework for higherorder quantum theory, Proc. R. Soc. A 475, 20180706 (2019).

[13] K. Życzkowski, Quartic quantum theory: an extension of the standard quantum mechanics, J. Phys. A: Math. Theo. 41, 355302 (2008).

[14] G. Chiribella, G. M. D'Ariano, P. Perinotti, and B. Valiron, Beyond quantum computers, arXiv:0912.0195.

[15] O. Oreshkov, F. Costa, and Č. Brukner, Quantum correlations with no causal order, Nat. Commun. 3, 1092 (2012).

[16] P. Perinotti, Causal structures and the classification of higher order quantum computations, in Time in Physics (Springer, 2017), pp. 103-127.

[17] E. Castro-Ruiz, F. Giacomini, and Č. Brukner, Dynamics of Quantum Causal Structures, Phys. Rev. X 8, 011047 (2018).

[18] D. Rosset, F. Buscemi, and Y.-C. Liang, Resource Theory of Quantum Memories and their Faithful Verification with Minimal Assumptions, Phys. Rev. X 8, 021033 (2018).

[19] D. Ebler, S. Salek, and G. Chiribella, Enhanced Communication with the Assistance of Indefinite Causal Order, Phys. Rev. Lett. 120, 120502 (2018).

[20] G. Gour and A. Winter, How to Quantify a Dynamical Quantum Resource, Phys. Rev. Lett. 123, 150401 (2019).

[21] X. Wang and M. M. Wilde, Resource theory of asymmetric distinguishability for quantum channels, Phys. Rev. Res. 1, 033169 (2019).

[22] J. Xu, Coherence of quantum channels, Phys. Rev. A 100, 052311 (2019)

[23] T. Theurer, D. Egloff, L. Zhang, and M. B. Plenio, Quantifying Operations with an Application to Coherence, Phys. Rev. Lett. 122, 190405 (2019).

[24] Y. Liu and X. Yuan, Operational resource theory of quantum channels, Phys. Rev. Res. 2, 012035(R) (2020).
[25] R. Takagi, K. Wang, and M. Hayashi, Application of the Resource Theory of Channels to Communication Scenarios, Phys. Rev. Lett. 124, 120502 (2020).

[26] H. Kristjánsson, G. Chiribella, S. Salek, D. Ebler, and M. Wilson, Resource theories of communication, New J. Phys. 22, 073014 (2020)

[27] G. Saxena, E. Chitambar, and G. Gour, Dynamical resource theory of quantum coherence, Phys. Rev. Res. 2, 023298 (2020).

[28] A. Bisio, G. Chiribella, G. M. D'Ariano, S. Facchini, and P. Perinotti, Optimal Quantum Tomography of States, Measurements, and Transformations, Phys. Rev. Lett. 102, 010404 (2009).

[29] G. Chiribella, G. M. D'Ariano, and P. Perinotti, Optimal Cloning of Unitary Transformation, Phys. Rev. Lett. 101, 180504 (2008).

[30] A. Bisio, G. M. D’Ariano, P. Perinotti, and M. Sedlák, Cloning of a quantum measurement, Phys. Rev. A 84, 042330 (2011).

[31] A. Bisio, G. Chiribella, G. M. D’Ariano, S. Facchini, and P. Perinotti, Optimal quantum learning of a unitary transformation, Phys. Rev. A 81, 032324 (2010).

[32] M. Sedlák and M. Ziman, Probabilistic storage and retrieval of qubit phase gates, Phys. Rev. A 102, 032618 (2020).

[33] G. Chiribella and D. Ebler, Optimal quantum networks and one-shot entropies, New J. Phys. 18, 093053 (2016).

[34] J. Miyazaki, A. Soeda, and M. Murao, Complex conjugation supermap of unitary quantum maps and its universal implementation protocol, Phys. Rev. Res. 1, 013007 (2019).

[35] M. T. Quintino, Q. Dong, A. Shimbo, A. Soeda, and M. Murao, Probabilistic exact universal quantum circuits for transforming unitary operations, Phys. Rev. A 100, 062339 (2019).

[36] G. Gour and C. M. Scandolo, Dynamical Entanglement, Phys. Rev. Lett. 125, 180505 (2020).

[37] S. Chen and E. Chitambar, Entanglement-breaking superchannels, Quantum 4, 299 (2020).

[38] K. Ben Dana, M. García Díaz, M. Mejatty, and A. Winter, Resource theory of coherence: Beyond states, Phys. Rev. A 95, 062327 (2017).

[39] K. Kraus, A. Böhm, J. D. Dollard, and W. H. Wootters, States, effects, and operations: fundamental notions of quantum theory. lectures in mathematical physics at the university of texas at austin, Lect. Notes Phys. 190 (1983).

[40] T. Heinosaari and M. Ziman, The Mathematical Language of Quantum Theory: from Uncertainty to Entanglement (Cambridge University Press, Cambridge, UK, 2011).

[41] J. Schwinger, The theory of quantized fields, Phys. Rev. 82, 914 (1951).

[42] G. Lüders, On the equivalence of invariance under time reversal and under particle-antiparticle conjugation for relativistic field theories, Dan. Mat. Fys. Medd. 28, 1 (1954).

[43] W. Pauli, in Niels Bohr and the Development of Physics (McGraw-Hill, New York, 1955), pp. 30-51.

[44] S. D. Bartlett, T. Rudolph, and R. W. Spekkens, Reference frames, superselection rules, and quantum information, Rev. Mod. Phys. 79, 555 (2007).

[45] E. Aurell, J. Zakrzewski, and K. Życzkowski, Time reversals of irreversible quantum maps, J. Phys. A: Math. Theor. 48, 38FT01 (2015).

[46] V. Karimipour, F. Benatti, and R. Floreanini, Quasi inversion of qubit channels, Phys. Rev. A 101, 032109 (2020). 
[47] F. Shahbeigi, K. Sadri, M. Moradi, K. Życzkowski, and V. Karimipour, Quasi-inversion of quantum and classical channels in finite dimensions, arXiv:2104.06062.

[48] Y. Aharonov, P. G. Bergmann, and J. L. Lebowitz, Time symmetry in the quantum process of measurement, Phys. Rev. 134, B1410 (1964).

[49] B. Reznik and Y. Aharonov, Time-symmetric formulation of quantum mechanics, Phys. Rev. A 52, 2538 (1995).

[50] Y. Aharonov, S. Popescu, and J. Tollaksen, A time-symmetric formulation of quantum mechanics, Phys. Today 63(11), 27 (2010).

[51] B. Coecke and R. Lal, Time Asymmetry of Probabilities Versus Relativistic Causal Structure: An Arrow of Time, Phys. Rev. Lett. 108, 200403 (2012).

[52] O. Oreshkov and N. J. Cerf, Operational formulation of time reversal in quantum theory, Nat. Phys. 11, 853 (2015).

[53] M. S. Leifer and M. F. Pusey, Is a time-symmetric interpretation of quantum theory possible without retrocausality? Proc. R. Soc. A 473, 20160607 (2017).

[54] B. Coecke, S. Gogioso, and J. H. Selby, The time-reverse of any causal theory is eternal noise, arXiv:1711.05511.

[55] A. Di Biagio, P. Donà, and C. Rovelli, Quantum information and the arrow of time, arXiv:2010.05734.

[56] G. Chiribella and Z. Liu, Quantum operations with indefinite time direction, arXiv:2012.03859.

[57] L. Hardy, Time symmetry in operational theories, arXiv:2104.00071.

[58] R. B. Griffiths, Consistent Quantum Theory (Cambridge University Press, Cambridge, UK, 2003).

[59] G. Chiribella, G. M. D’Ariano, and P. Perinotti, Probabilistic theories with purification, Phys. Rev. A 81, 062348 (2010).

[60] G. Chiribella, G. M. D'Ariano, and P. Perinotti, Informational derivation of quantum theory, Phys. Rev. A 84, 012311 (2011).

[61] G. Chiribella, G. M. D’Ariano, and P. Perinotti, "Quantum from principles, in Quantum Theory: Informational Foundations and Foils (Springer, 2016), pp. 171-221.

[62] G. M. D’Ariano, G. Chiribella, and P. Perinotti, Quantum Theory from First Principles: an Informational Approach (Cambridge University Press, Cambridge, UK, 2017).

[63] M. Horodecki and J. Oppenheim, Fundamental limitations for quantum and nanoscale thermodynamics, Nat. Commun. 4, 2059 (2013).

[64] G. Gour, M. P. Müller, V. Narasimhachar, R. W. Spekkens, and N. Y. Halpern, The resource theory of informational nonequilibrium in thermodynamics, Phys. Rep. 583, 1 (2015).

[65] G. Chiribella and C. M. Scandolo, Microcanonical thermodynamics in general physical theories, New J. Phys. 19, 123043 (2017).

[66] A. Uhlmann, Anti- (conjugate) linearity, Sci. China 59, 630301 (2016)

[67] A. S. Holevo, Quantum Systems, Channels, Information: a Mathematical Introduction (Walter de Gruyter, Berlin/Boston, 2012), Vol. 16.

[68] V. Cappellini, H.-J. Sommers, and K. Życzkowski, Subnormalized states and trace-nonincreasing maps, J. Math. Phys. 48, 052110 (2007).

[69] A. S. Holevo and R. F. Werner, Evaluating capacities of bosonic gaussian channels, Phys. Rev. A 63, 032312 (2001).
[70] Y. Yang, G. Chiribella, and Q. Hu, Units of rotational information, New J. Phys. 19, 123003 (2017).

[71] L. J. Landau and R. F. Streater, On Birkhoff's theorem for doubly stochastic completely positive maps of matrix algebras, Linear Algebra Appl. 193, 107 (1993).

[72] C. B. Mendl and M. M. Wolf, Unital quantum channelsconvex structure and revivals of birkhoff's theorem, Commun. Math. Phys. 289, 1057 (2009).

[73] D. Petz, Sufficiency of channels over von neumann algebras, Q. J. Math. 39, 97 (1988).

[74] H. Barnum and E. Knill, Reversing quantum dynamics with near-optimal quantum and classical fidelity, J. Math. Phys. 43, 2097 (2002).

[75] P. Hayden, R. Jozsa, D. Petz, and A. Winter, Structure of states which satisfy strong subadditivity of quantum entropy with equality, Commun. Math. Phys. 246, 359 (2004).

[76] H. K. Ng and P. Mandayam, Simple approach to approximate quantum error correction based on the transpose channel, Phys. Rev. A 81, 062342 (2010).

[77] O. Fawzi and R. Renner, Quantum conditional mutual information and approximate Markov chains, Commun. Math. Phys. 340, 575 (2015).

[78] S. Beigi, N. Datta, and F. Leditzky, Decoding quantum information via the petz recovery map, J. Math. Phys. 57, 082203 (2016).

[79] M. Junge, R. Renner, D. Sutter, M. M. Wilde, and A. Winter, Universal recovery maps and approximate sufficiency of quantum relative entropy, in Annales Henri Poincaré (Springer, 2018), Vol. 19, pp. 2955-2978.

[80] M.-D. Choi, Completely positive linear maps on complex matrices, Lin. Alg. Appl. 10, 285 (1975).

[81] I. Bengtsson and K. Życzkowski, Geometry of Quantum States, 2nd ed. (Cambridge University Press, 2017).

[82] H.-D. Doebner and G. A. Goldin, On a general nonlinear Schrödinger equation admitting diffusion currents, Phys. Lett. A 162, 397 (1992).

[83] H.-D. Doebner, G. A. Goldin, and P. Nattermann, Gauge transformations in quantum mechanics and the unification of nonlinear Schrödinger equations, J. Math. Phys. 40, 49 (1999).

[84] G. A. Goldin, Nonlinear quantum mechanics: Results and open questions, Phys. At. Nucl. 71, 884 (2008).

[85] E. G. Cavalcanti, N. C. Menicucci, and J. L. Pienaar, The preparation problem in nonlinear extensions of quantum theory, arXiv:1206.2725.

[86] G. E. Crooks, Quantum operation time reversal, Phys. Rev. A 77, 034101 (2008).

[87] S. Saunders, J. Barrett, A. Kent, and D. Wallace, Many worlds?: Everett, Quantum Theory, and Reality (Oxford University Press, 2010).

[88] A. Uhlmann, The "transition probability" in the space of a *-algebra, Rep. Math. Phys. 9, 273 (1976).

[89] R. Jozsa, Fidelity for mixed quantum states, J. Mod. Opt. 41, 2315 (1994)

[90] A. Chefles, R. Jozsa, and A. Winter, On the existence of physical transformations between sets of quantum states, Int. J. Quantum. Inform. 2, 11 (2004).

[91] A. S. Holevo, Probabilistic and Statistical Aspects of Quantum Theory (Springer Science \& Business Media, 2011), Vol. 1. 
[92] D. W. Leung, Towards robust quantum computation, arXiv:cs/0012017 [cs.CC].

[93] G. M. D'Ariano and P. Lo Presti, Quantum Tomography for Measuring Experimentally the Matrix Elements of an Arbitrary Quantum Operation, Phys. Rev. Lett. 86, 4195 (2001).

[94] P. Arrighi and C. Patricot, On quantum operations as quantum states, Ann. Phys. 311, 26 (2004).

[95] K. Życzkowski and I. Bengtsson, On duality between quantum states and quantum maps, Open Syst. Inf. Dyn. 11, 3 (2004).

[96] S. Abramsky and B. Coecke, A categorical semantics of quantum protocols, in Proceedings of the 19th Annual IEEE Symposium on Logic in Computer Science, 2004 (IEEE, 2004), pp. 415-425.
[97] A. Jamiołkowski, Linear transformations which preserve trace and positive semi-definiteness of operators, Rep. Math. Phys. 3, 275 (1972).

[98] G. Chiribella, G. M. D'Ariano, and P. Perinotti, Memory Effects in Quantum Channel Discrimination, Phys. Rev. Lett. 101, 180501 (2008).

[99] G. Chiribella, Distinguishability and copiability of programs in general process theories, Int. J. Software Inform. 8, 209 (2014).

[100] G. Gour, Comparison of quantum channels by superchannels, IEEE Trans. Inf. Theory 65, 5880 (2019).

[101] J. Burniston, M. Grabowecky, C. M. Scandolo, G. Chiribella, and G. Gour, Necessary and sufficient conditions on measurements of quantum channels, Proc. R. Soc. A 476, 20190832 (2020). 